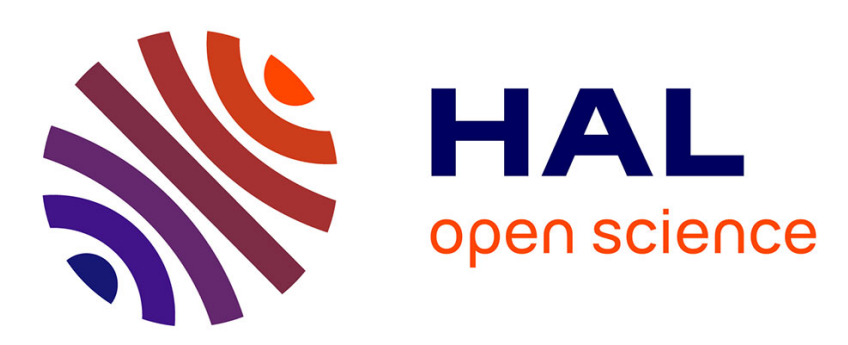

\title{
Numerical modeling of three-dimensional open elastic waveguides combining semi-analytical finite element and perflectly matched layer methods
}

Khac-Long Nguyen, Fabien Treyssede, Christophe Hazard

\section{- To cite this version:}

Khac-Long Nguyen, Fabien Treyssede, Christophe Hazard. Numerical modeling of three-dimensional open elastic waveguides combining semi-analytical finite element and perflectly matched layer methods. Journal of Sound and Vibration, 2015, 344, pp.158-178. 10.1016/j.jsv.2014.12.032 . hal-01346096v2

\section{HAL Id: hal-01346096 \\ https://hal.science/hal-01346096v2}

Submitted on 10 Feb 2017

HAL is a multi-disciplinary open access archive for the deposit and dissemination of scientific research documents, whether they are published or not. The documents may come from teaching and research institutions in France or abroad, or from public or private research centers.
L'archive ouverte pluridisciplinaire HAL, est destinée au dépôt et à la diffusion de documents scientifiques de niveau recherche, publiés ou non, émanant des établissements d'enseignement et de recherche français ou étrangers, des laboratoires publics ou privés. 


\title{
Numerical modeling of three-dimensional open elastic waveguides combining semi-analytical finite element and perfectly matched layer methods
}

\author{
K. L. Nguyen ${ }^{\mathrm{a}, *}$, F. Treyssède ${ }^{\mathrm{a}}$, C. Hazard ${ }^{\mathrm{b}}$ \\ ${ }^{a}$ LUNAM Université, IFSTTAR, GERS, F-44344 Bouguenais, France \\ ${ }^{b}$ ENSTA-UMA, POEMS, 828 Boulevard des Maréchaux, 91120 Palaiseau, France
}

\begin{abstract}
Among the numerous techniques of non destructive evaluation, elastic guided waves are of particular interest to evaluate defects inside industrial and civil elongated structures owing to their ability to propagate over long distances. However for guiding structures buried in large solid media, waves can be strongly attenuated along the guide axis due to the energy radiation into the surrounding medium, usually considered as unbounded. Hence, searching the less attenuated modes become necessary in order to maximize the inspection distance. In the numerical modeling of embedded waveguides, the main difficulty is to account for the unbounded section. This paper presents a numerical approach combining a semi-analytical finite element method and a perfectly matched layer (PML) technique to compute the so-called trapped and leaky modes in three-dimensional embedded elastic waveguides of arbitrary cross-section. Two kinds of PML, namely the Cartesian PML and the radial PML, are considered. In order to understand the various spectral objects obtained by the method, the PML parameters effects upon the eigenvalue spectrum are highlighted through analytical studies and numerical experiments. Then, dispersion curves are computed for test cases taken from the literature in order to validate the approach.
\end{abstract}

Keywords:

\section{Introduction}

Among the numerous techniques of non destructive evaluation (NDE), elastic guided waves are of particular interest to evaluate defects inside industrial and civil elongated structures due to their ability to propagate over long distances. Two categories of waveguides can be distinguished: closed waveguides (guides in vacuum) and open waveguides (embedded waveguides).

In closed waveguides, waves can propagate along the guide axis without attenuation. However in practice, guides are often embedded in large solid media that can be considered as unbounded. In this case, waveguides are called open because of the energy radiation into the surrounding medium. Three kinds of wave modes can occur in open waveguides: radiation modes, trapped modes and leaky ones. Their characteristics are briefly recalled in the following paragraphs. These modes are obtained by assuming a dependence of wave fields in $\mathrm{e}^{\mathrm{i}(k z-\omega t)}$, where $k$ is the axial wavenumber, $\omega$ is the angular frequency and $z$ is the coordinate along the waveguide axis. The following dispersion relations holds: $k^{2}+k_{l / s}^{2}=\omega^{2} / c_{l / s}^{2}$, where $c_{l}$ and $c_{s}$ are the longitudinal and shear wave speeds, $k_{l}$ and $k_{s}$ denote the longitudinal and shear transverse wavenumbers of the unbounded medium respectively.

Radiation modes are standing waves in the transverse directions and can be either oscillating or evanescent in the longitudinal direction, i.e. $k_{l / s} \in \mathbb{R}$ and $k$ is real or pure imaginary. They constitute a continuous spectrum [1, 2], resulting from the unbounded nature of the problem. Resonating mainly in the surrounding medium, radiation modes are of little interest for the NDE of elongated structures.

Conversely, trapped modes are of particular interest. These modes exponentially decay in the transverse directions $\left(k_{l / s} \in i \mathbb{R}\right)$ and propagate along the axis without attenuation $(k \in \mathbb{R})$ in non-dissipative waveguides. Their

\footnotetext{
${ }^{*}$ Corresponding author

Email address: khac-long.nguyen@ifsttar.fr (K. L. Nguyen)

Preprint submitted to Journal of Sound and Vibration
} 
energy is confined into the core of waveguides without energy leakage into the surrounding medium allowing long inspection distances. Nevertheless, trapped modes do not always occur. For scalar open waveguides (characterized by a scalar field such as the acoustic pressure or the SH wave displacement), trapped modes exist only if the bulk velocity in the core is lower than in the surrounding medium [3]. In the elastic case, both compressional and shear bulk waves occur and, unless Stoneley waves are allowed on the interface between materials, no trapped modes are present when the shear velocity is faster in the core [4,5]. Unfortunately, such a configuration is often encountered in civil structures because the guiding structures are usually embedded in soft solid media such as concrete, cement or grout for instance.

As opposed to trapped modes, leaky modes always exist in open waveguides. Their energy leakage into the surrounding medium yields attenuation along the guide axis $(k \in \mathbb{C} / \mathbb{R}, \operatorname{Im}(k)$ being positive for positivegoing modes and negative for negative-going ones), which can strongly limit their propagation distance. An unusual behavior of leaky modes is that, while decaying along the axis, their amplitudes increase in the transverse directions. This feature is well-known in electromagnetism [3,6] and has sometimes been mentioned in elastodynamics $[7,8,9,10,11]$.

From a mathematical point of view, leaky modes are not spectral objects because they belong to the forbidden Riemann sheet $[3,12]$. They constitute a discrete set which is not part of the complete set constituted by the continuum of radiation modes and the discrete set of trapped modes [1,2]. The mathematical characterization of leaky modes requires a complicated analytical continuation [13] which is out of scope here. From a physical point of view, leaky modes can constitute a good approximation of the continuum of radiation modes in the excited field over a restricted area, near the core region [6]. Through the imaginary part of their axial wavenumbers, leaky modes allow to directly evaluate the ability of waves to propagate far away along the axis. With radiation modes, such an information is hidden into the continuum. Therefore, an accurate determination of leaky modes is essential for the NDE of embedded waveguides in order to find modes and frequencies of lowest attenuation.

In the literature, many researches have been conducted on embedded waveguides based on analytical approaches $[14,15]$. Analytical models are mainly based on transfer matrix or global matrix methods $[14,15]$ and allow to plot the dispersion curves of solid embedded waveguides. Methods based on Debye series have also been proposed in Ref. [16]. Yet, these techniques are limited to simple geometries such as plates and cylinders $[17,18,19]$.

The modeling of more complex geometries usually relies on numerical approaches. A powerful technique is the so-called Semi-Analytical Finite Element (SAFE) method [20, 21, 22, 23], which restricts the FE discretization on transverse directions only. The SAFE method has been mainly used for the simulation of closed waveguides. The numerical modeling of open waveguides encounters two difficulties: the cross-section is unbounded and the amplitude of leaky modes transversely increases. In order to overcome these difficulties, the SAFE method must be associated with other techniques.

A simple numerical procedure is the absorbing layer (AL) method proposed in Refs. [24, 25], which consists in creating artificial viscoelastic layers in the surrounding medium for absorbing waves. Instead of using artificial layers, Mazzotti et al. have recently combined the boundary element method (BEM) with the SAFE method to model three-dimensional elastic waveguides embedded in a solid [26] or in a fluid [27]. The BEM expresses the solution in the exterior domain by integral formula on the core boundary. Analogously to the SAFE-BEM method, Hayashi et al. [28] have proposed a formulation for open plate waveguides. An alternative technique is the perfectly matched layer (PML) method, which consists in analytically extending real coordinates of physical equations into the complex ones. The SAFE-PML method has already been applied to scalar wave problems [29, 30, 31]. More recently, the authors have presented this method to model open solid plate waveguides [32, 33] (one-dimensional modal problems).

Among these three techniques, the SAFE-AL method is the simplest to implement as it does not require specific programming in existing codes. However, the AL artificial viscoelasticity must be slowly growing in order to minimize reflection so that large layers can be required in practice, hence increasing the computational cost. Compared to AL, the PML thickness is expected to be significantly reduced. Theoretically, the PML can strongly attenuate waves without artificial reflection thanks to the analytical extension of coordinates (perfectly matched property). Contrary to SAFE-AL and SAFE-PML methods, the SAFE-BEM method avoids the discretization of the unbounded medium, which significantly reduces the computational domain. Yet, the SAFE-BEM eigenproblem is highly non linear and difficult to solve. To avoid the eigenproblem non-linearity with SAFE-BEM 


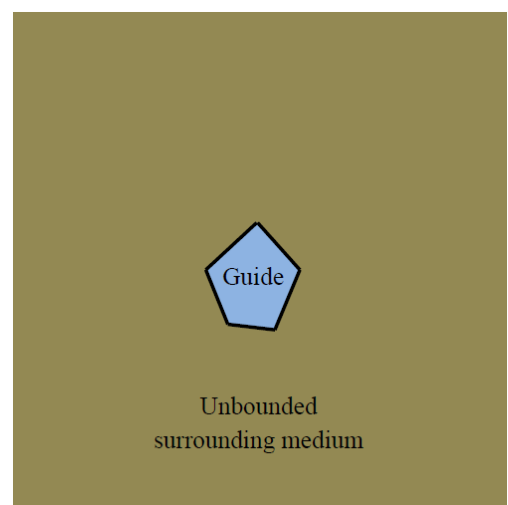

(a)

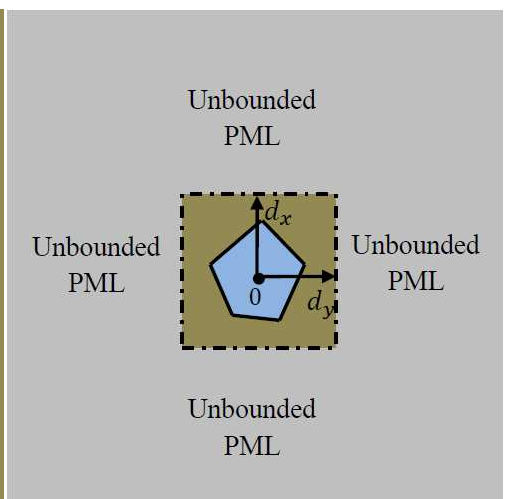

(b)

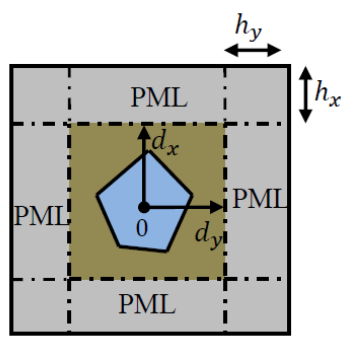

(c)

Figure 1: (a) Arbitrary cross-section of an open waveguide, (b) introduction of Cartesian PML in the surrounding medium, (c) PML truncation.

methods, some modified formulations have been proposed. Hayashi et al. [28] have successfully transformed the non linear eigenproblem into a linear one for the special case of a surrounding fluid (scalar waves). Gravenkamp et al. [34] have proposed a simplified boundary condition, namely dashpot boundary condition, which amounts to neglect transverse wavenumbers in the exact radiation condition. This dashpot condition is usually no longer accurate for low frequency or for low contrast of acoustic impedance. Unlike the SAFE-BEM approach, both the SAFE-AL and SAFE-PML methods yield a linear eigenvalue problem.

In this paper, the SAFE-PML technique is proposed as an alternative to SAFE-AL and SAFE-BEM methods to compute modes in three-dimensional elastic waveguides of arbitrary cross-section embedded in an unbounded solid matrix. Two kinds of PML, Cartesian and radial, are considered. This yields two formulations referred to as the SAFE-Cartesian PML (SAFE-CPML) method and the SAFE-radial PML (SAFE-RPML) method. It is pointed out that both kinds of PML have been analyzed mathematically for computing the acoustic resonances of open cavities $[35,36]$, which constitutes a problem close to that of the present paper. Note also that the SAFECPML formulation of this paper is indeed similar to the 2.5D displacement-based PML formulation recently proposed in Ref. [37] (yet in this reference, the discretized problem was not considered as an eigenvalue problem, but rather inverted by considering a source term for fixed transverse wavenumbers).

This paper focuses on the implementation and the validation of the SAFE-PML method. The comparative study between this technique and other techniques such as SAFE-AL or SAFE-BEM methods is out of scope of this paper and left for further studies.

The SAFE-CPML and SAFE-RPML formulations are presented in Secs. 2 and 3 respectively. In order to understand the various spectral objects obtained from these formulations and to clarify the effects of PML parameters, the eigenvalue spectrum is analyzed for each formulation based on analytical studies and numerical experiments. In Sec. 4, the computation of modal properties (group velocity, energy velocity and kinetic energy) is introduced and dispersion curves are computed for several test cases taken from the literature to validate both formulations.

\section{SAFE - CPML method}

\subsection{Initial formulation of $3 D$ elastodynamics}

One considers a three-dimensional waveguide $\tilde{\Omega}=\tilde{S} \times]-\infty,+\infty$ [. Linear elastic materials are assumed. The waveguide cross-section $\tilde{S}$ lies in the $(\tilde{x}, \tilde{y})$ plane. The tilde notation will be explained through the introduction of PML in Sec. 2.2. The time harmonic dependence is chosen as $\mathrm{e}^{-\mathrm{i} \omega t}$. The study is focused on eigenmodes. Acoustic sources and external forces are then discarded.

The variational formulation of the elastodynamic problem for the displacement field $\mathbf{u}$ is given by

$$
\int_{\tilde{\Omega}} \delta \tilde{\boldsymbol{\epsilon}}^{T} \tilde{\boldsymbol{\sigma}} \mathrm{d} \tilde{\Omega}-\omega^{2} \int_{\tilde{\Omega}} \tilde{\rho} \delta \tilde{\mathbf{u}}^{T} \tilde{\mathbf{u}} \mathrm{d} \tilde{\Omega}=0
$$


where $\mathrm{d} \tilde{\Omega}=\mathrm{d} \tilde{x} \mathrm{~d} \tilde{y} \mathrm{~d} z$. This formulation holds for any kinematically admissible displacement $\delta \tilde{\mathbf{u}}=\left[\delta \tilde{u}_{x} \delta \tilde{u}_{y} \delta \tilde{u}_{z}\right]^{T}$.

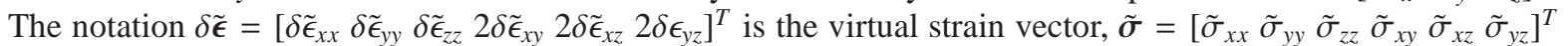
denotes the stress vector. The stress-strain relation is given by $\tilde{\boldsymbol{\sigma}}=\tilde{\mathbf{C}} \tilde{\boldsymbol{\epsilon}}$, where $\tilde{\mathbf{C}}$ is the matrix of material properties. $\tilde{\rho}$ is the material mass density. The superscript $T$ denotes the matrix transpose. We assume that $\tilde{\mathbf{C}}$ and $\tilde{\rho}$ depend only on the transverse coordinates $(\tilde{x}, \tilde{y})$, which means that we consider translationally invariant waveguides along the $z$ axis. Moreover we assume that the medium is homogeneous outside a bounded region which represents the core of the waveguide.

Separating transverse from axial derivatives, the strain-displacement relation can be written as follows:

$$
\tilde{\boldsymbol{\epsilon}}=\left(\mathbf{L}_{\tilde{S}}+\mathbf{L}_{z} \frac{\partial}{\partial z}\right) \tilde{\mathbf{u}}
$$

where $\mathbf{L}_{\tilde{S}}=\mathbf{L}_{x} \frac{\partial}{\partial \tilde{x}}+\mathbf{L}_{y} \frac{\partial}{\partial \tilde{y}}$ and

$$
\mathbf{L}_{x}=\left[\begin{array}{ccc}
1 & 0 & 0 \\
0 & 0 & 0 \\
0 & 0 & 0 \\
0 & 1 & 0 \\
0 & 0 & 1 \\
0 & 0 & 0
\end{array}\right], \mathbf{L}_{y}=\left[\begin{array}{ccc}
0 & 0 & 0 \\
0 & 1 & 0 \\
0 & 0 & 0 \\
1 & 0 & 0 \\
0 & 0 & 0 \\
0 & 0 & 1
\end{array}\right], \mathbf{L}_{z}=\left[\begin{array}{ccc}
0 & 0 & 0 \\
0 & 0 & 0 \\
0 & 0 & 1 \\
0 & 0 & 0 \\
1 & 0 & 0 \\
0 & 1 & 0
\end{array}\right] .
$$

$\mathbf{L}_{\tilde{S}}$ and $\mathbf{L}_{z} \partial / \partial z$ are the operators of derivatives with respect to the transverse directions $(\tilde{x}, \tilde{y})$ and the axis $z$ respectively.

\subsection{Combining SAFE and Cartesian PML techniques}

The SAFE method consists in assuming a harmonic axial dependence of fields and applying the FE method to the transverse directions. The problem is then reduced from three dimensions to the two dimensions of the waveguide cross-section. The SAFE method has been widely used for modeling closed waveguides (guides in vacuum), for which the cross-section is bounded (see for instance [20, 21, 22, 23]).

The modeling of open waveguides requires to combine the SAFE method with another technique due to the unbounded nature of the section. We assume that outside a possibly inhomogeneous region representing the core of the waveguide, the medium is homogeneous. The basic idea consists in closing the waveguide section by replacing the unbounded homogeneous region with a PML of finite thickness. As shown in Fig. 1, a PML is introduced along the Cartesian transverse coordinates in order to attenuate waves in the surrounding medium. By truncating the cross-section to a sufficiently large distance, the problem becomes closed and the SAFE method can be applied. In this case, $\tilde{S}$ denotes the truncated section including the PML.

The basic principle of PML can be readily understood in a one-dimensional situation. Consider for instance the case of a longitudinal wave traveling in the positive $\tilde{x}$ direction. Such a wave can be expressed as an exponential function $\exp \left(\mathrm{i} k_{l} \tilde{x}\right)$, which extends to an entire function for complex values of $\tilde{x}$. Hence, instead of considering real $\tilde{x}$, one can choose a particular path $\tilde{x}(x)$ in the complex plane parametrized by a real variable $x$ such that $\exp \left(\mathrm{i} k_{l} \tilde{x}(x)\right)$ decays exponentially as $x$ tends to $+\infty$. The same considerations apply in the $\tilde{y}$ direction or for a shear wave. The Cartesian PML method consists in extending the initial equilibrium equations to complex coordinates $\tilde{x}$ and $\tilde{y}$, properly parametrized to attenuate waves (the PML parametrization will be discussed in Sec. 2.4). Here we define

$$
\tilde{x}(x)=\int_{0}^{x} \gamma_{x}(\xi) \mathrm{d} \xi, \quad \tilde{y}(y)=\int_{0}^{y} \gamma_{y}(\xi) \mathrm{d} \xi
$$

where $\gamma_{x}(x), \gamma_{y}(y)$ are complex functions satisfying

- $\gamma_{x}(x)=1$ for $|x| \leq d_{x} ; \gamma_{y}(y)=1$ for $|y| \leq d_{y}$,

- $\operatorname{Im}\left\{\gamma_{x}\right\}>0$ for $|x|>d_{x} ; \operatorname{Im}\left\{\gamma_{y}\right\}>0$ for $|y|>d_{y}$. 
$d_{x}$ and $d_{y}$ are positive parameters chosen such that the rectangle $\left[-d_{x},+d_{x}\right] \times\left[-d_{y},+d_{y}\right]$ contains the inhomogeneous part of the medium. Thus $\tilde{x}$ and $\tilde{y}$ become non-real in the homogeneous surrounding medium.

Since waves are attenuated, the PML can be truncated at a finite distance. We denote by $h_{x}$ and $h_{y}$ the PML thicknesses in the $x$ and $y$ directions respectively (see Fig. 1c). Thus, in the $(x, y)$ plane, the truncated cross-section including the PML is the rectangle of half-thicknesses $d_{x}+h_{x}$ and $d_{y}+h_{y}$. On the exterior boundary of the PML, the boundary condition can be arbitrarily chosen (usually of Dirichlet type).

From Eq. (4), the change of variables $\tilde{x} \mapsto x, \tilde{y} \mapsto y$ yields for any function $\tilde{f}$ :

$$
\frac{\partial \tilde{f}}{\partial \tilde{x}}=\frac{1}{\gamma_{x}} \frac{\partial f}{\partial x}, \frac{\partial \tilde{f}}{\partial \tilde{y}}=\frac{1}{\gamma_{y}} \frac{\partial f}{\partial y}, \mathrm{~d} \tilde{x}=\gamma_{x} \mathrm{~d} x, \mathrm{~d} \tilde{y}=\gamma_{y} \mathrm{~d} y
$$

where $\tilde{f}(\tilde{x}(x), \tilde{y}(y), z)=f(x, y, z)$.

Applying this change of variable to Eq. (2) leaves the operator $\mathbf{L}_{z}$ unchanged while the operator $\mathbf{L}_{\tilde{S}}$ has to be replaced with

$$
\mathbf{L}_{S}=\frac{1}{\gamma_{x}} \mathbf{L}_{x} \frac{\partial}{\partial x}+\frac{1}{\gamma_{y}} \mathbf{L}_{y} \frac{\partial}{\partial y} .
$$

Now applying the SAFE method, the displacement $\mathbf{u}$ and the virtual displacement $\delta \mathbf{u}$ are expressed on one element $e$ as follows:

$$
\mathbf{u}(x, y, z)=\mathbf{N}^{e}(x, y) \mathbf{U}^{e} \mathrm{e}^{\mathrm{i} k z}, \delta \mathbf{u}(x, y, z)=\mathbf{N}^{e}(x, y) \delta \mathbf{U}^{e} \mathrm{e}^{-\mathrm{i} k z}
$$

where $k$ is the axial wavenumber, $\mathbf{U}^{e}$ is the displacement vector and $\mathbf{N}^{e}$ is the matrix of interpolating functions on the element $e$.

Replacing the axial derivative $\partial / \partial z$ of the trial and test functions with products by $+\mathrm{i} k$ and $-\mathrm{i} k$ respectively, the formulation (1) is reduced from three dimensions $(x, y, z)$ to a bidimensional problem written in the transverse directions $(x, y)$. The strain-displacement relation becomes

$$
\boldsymbol{\epsilon}=\left(\mathbf{L}_{S}+\mathrm{i} k \mathbf{L}_{z}\right) \mathbf{N}^{e} \mathbf{U}^{e} \mathrm{e}^{\mathrm{i} k z} .
$$

The FE discretization of the truncated cross-section finally yields

$$
\left\{\mathbf{K}_{1}-\omega^{2} \mathbf{M}+\mathrm{i} k\left(\mathbf{K}_{2}-\mathbf{K}_{2}^{T}\right)+k^{2} \mathbf{K}_{3}\right\} \mathbf{U}=\mathbf{0}
$$

with the elementary matrices:

$$
\begin{aligned}
& \mathbf{K}_{1}^{e}=\int_{e} \mathbf{N}^{e T} \mathbf{L}_{S}^{T} \mathbf{C L}_{S} \mathbf{N}^{e} \gamma_{x} \gamma_{y} \mathrm{~d} x \mathrm{~d} y, \mathbf{K}_{2}^{e}=\int_{e} \mathbf{N}^{e T} \mathbf{L}_{S}^{T} \mathbf{C} \mathbf{L}_{z} \boldsymbol{N}^{e} \gamma_{x} \gamma_{y} \mathrm{~d} x \mathrm{~d} y \\
& \mathbf{K}_{3}^{e}=\int_{e} \mathbf{N}^{e T} \mathbf{L}_{z}^{T} \mathbf{C} \mathbf{L}_{z} \boldsymbol{N}^{e} \gamma_{x} \gamma_{y} \mathrm{~d} x \mathrm{~d} y, \mathbf{M}^{e}=\int_{e} \rho \mathbf{N}^{e T} \mathbf{N}^{e} \gamma_{x} \gamma_{y} \mathrm{~d} x \mathrm{~d} y .
\end{aligned}
$$

Note that the SAFE-CPML matrices are complex due to the functions $\gamma_{x}$ and $\gamma_{y}$ in the integrands.

\subsection{Linear eigenvalue problem}

Given the frequency $\omega$, the formulation (9) is quadratic with respect to $k$, which can be linearized as $[33,38]$

$$
(\mathbf{A}-k \mathbf{B}) \hat{\mathbf{U}}=0
$$

with

$$
\mathbf{A}=\left[\begin{array}{cc}
\mathbf{0} & \mathbf{I} \\
-\left(\mathbf{K}_{1}-\omega^{2} \mathbf{M}\right) & -\mathrm{i}\left(\mathbf{K}_{2}-\mathbf{K}_{2}^{T}\right)
\end{array}\right], \quad \mathbf{B}=\left[\begin{array}{cc}
\mathbf{I} & \mathbf{0} \\
\mathbf{0} & \mathbf{K}_{3}
\end{array}\right], \hat{\mathbf{U}}=\left[\begin{array}{c}
\mathbf{U} \\
k \mathbf{U}
\end{array}\right] .
$$

The symmetry of $\mathbf{K}_{1}, \mathbf{K}_{3}$ and $\mathbf{M}$ implies that if $k$ is an eigenvalue of (9), then $-k$ is also an eigenvalue. Thus, the eigenspectrum includes two families of solutions, $\left(k_{j}, \mathbf{U}_{j}^{+}\right)$and $\left(-k_{j}, \mathbf{U}_{j}^{-}\right),(j=1, \ldots, n)$ representing $n$ positive-going and $n$ negative-going waves.

In the presence of PML, $\mathbf{K}_{1}, \mathbf{K}_{2}, \mathbf{K}_{3}$ and $\mathbf{M}$ are complex. $\mathbf{A}$ and $\mathbf{B}$ are not Hermitian, which somewhat complicates the numerical treatment of the eigensystem (10). As outlined in Ref. [35, 30], the non-hermitian character of matrices may yield spurious eigenvalues which are associated with large values of the norm of resolvent. This problem can be reduced by setting the PML not too far from the core.

In this paper, the ARPACK library [39] is used for solving the eigensystem (10). This library is based on the implicitly restarted Arnoldi method. For each frequency, a specified number of eigenvalues is looked for around a user-defined shift. 


\subsection{PML absorbing functions}

In each direction $x$ and $y$, the PML depends on three user-defined parameters: the position of the interface $\left(d_{x}, d_{y}\right)$, the thickness $\left(h_{x}, h_{y}\right)$ and the absorbing function $\left(\gamma_{x}, \gamma_{y}\right)$. For a given interface position and PML thickness, the optimal choice of absorbing functions is crucial for maximizing the attenuation of waves with minimal reflections.

In scattering problems (source problems), the PML function is usually frequency dependent $[40,41]$ and chosen as: $\gamma_{x}(x)=1+\mathrm{i} \sigma_{x}(x) / \omega$ where $\sigma_{x}(x)$ is a continuous function, parabolic inside the PML region (a similar expression holds for $\left.\gamma_{y}(y)\right)$. In modal problems, the proper choice of PML functions is slightly different.

As mentioned in the preceding section, if we consider a longitudinal or shear wave $\exp \left(\mathrm{i} k_{l / s} \tilde{x}\right)$ for real $\tilde{x}$ (where $k_{l}$ and $k_{s}$ denote the transverse longitudinal and shear wavenumbers), the effect of an infinite PML in the positive $x$ direction is to transform this function into an exponentially decaying function $\exp \left(\mathrm{i} k_{l / s} \tilde{x}(x)\right)$ as $x$ tends to $+\infty$. From (4), it is easily seen that the total attenuation across a layer of finite thickness $h_{x}$ is given by

$$
\exp \left(-\operatorname{Im}\left(k_{l / s} \hat{\gamma}_{x} h_{x}\right)\right)=\exp \left(-\left|k_{l / s}\right|\left|\hat{\gamma}_{x}\right| h_{x} \sin \left(\arg k_{l / s}+\arg \hat{\gamma}_{x}\right)\right)
$$

where $\hat{\gamma}_{x}$ denotes the average values of $\gamma_{x}$ in the layer :

$$
\hat{\gamma}_{x}=\frac{1}{h_{x}} \int_{d_{x}}^{d_{x}+h_{x}} \gamma_{x}(\xi) \mathrm{d} \xi .
$$

One recalls that leaky modes decay along the axial direction $z(\operatorname{Im} k>0)$ but grow in the transverse direction, i.e. $\operatorname{Im} k_{l / s}<0$ or equivalently $\arg k_{l / s}<0$. From Eq. (12), leaky waves can be attenuated by the PML if $\hat{\gamma}_{x}$ is such that $\arg k_{l / s}>-\arg \hat{\gamma}_{x}$ (in this paper, arg denotes the principal argument and lies in the interval $\left.]-\pi,+\pi\right]$ ). Hence, increasing $\arg \hat{\gamma}_{x}$ will enlarge the region of the complex plane where leaky modes can be computed. Increasing $\left|\hat{\gamma}_{x}\right| h_{x}$ will increase the PML absorption.

For trapped modes, waves propagate without axial attenuation $(\operatorname{Im} k=0)$ and exponentially decay in the transverse direction so that: $\operatorname{Re} k_{l / s}=0$ and $\operatorname{Im} k_{l / s}>0$, or equivalently $\arg k_{l / s}=\pi / 2$. In the presence of PML, trapped waves will thus remain decaying if $\arg \hat{\gamma}_{x}<\pi / 2$. The PML will be able to enhance the natural decay of trapped modes if $\operatorname{Im}\left(k_{l / s} \hat{\gamma}_{x}\right)>\operatorname{Im} k_{l / s}$, or equivalently $\operatorname{Re} \hat{\gamma}_{x}>1$.

Note that $\left|\hat{\gamma}_{x}\right|$ cannot be too high in practice for a given $h_{x}$, otherwise waves will attenuate too fast to be well approximated by the FE discretization. This phenomenon is well-known in the PML literature [40].

For a PML introduced in the $y$-direction, the above considerations also holds with $\hat{\gamma}_{y}$ defined by

$$
\hat{\gamma}_{y}=\frac{1}{h_{y}} \int_{d_{y}}^{d_{y}+h_{y}} \gamma_{y}(\xi) \mathrm{d} \xi
$$

For open waveguide modal problems, the PML functions have been usually set to a constant complex value [30, $42,43,44]$, yielding a discontinuity at the PML interface. However a smooth profile can improve the accuracy of modes, as recently shown in Refs. $[32,33]$. In this paper, a parabolic function is set for both the real and the imaginary parts of $\gamma_{x}$ and $\gamma_{y}$ :

$$
\gamma_{x}(x)=\left\{\begin{array}{ll}
1 & \text { if } \quad|x| \leq d_{x} \\
1+3\left(\hat{\gamma}_{x}-1\right)\left(\frac{|x|-d_{x}}{h_{x}}\right)^{2} & \text { if } \quad|x|>d_{x}
\end{array}, \gamma_{y}(y)=\left\{\begin{array}{lll}
1 & \text { if } & |y| \leq d_{y} \\
1+3\left(\hat{\gamma}_{y}-1\right)\left(\frac{|y|-d_{y}}{h_{y}}\right)^{2} & \text { if } \quad|y|>d_{y}
\end{array} .\right.\right.
$$

$\hat{\gamma}_{x}$ and $\hat{\gamma}_{y}$ quantify the PML absorption. Note that the PML functions $\gamma_{x}$ and $\gamma_{y}$ are independent of $\omega$, which avoids the calculation of SAFE-CPML matrices at each frequency.

\subsection{Eigenspectrum}

The goal of this subsection is to get a better understanding of the influence of each PML parameter upon each type of modes. First, the analytical solution of a homogeneous medium is derived and compared with numerical results obtained with the SAFE-CPML method. Second, a more complex case corresponding to a steel cylinder buried in a solid medium is considered. Numerical experiments are performed to understand how the Cartesian PML acts on the eigenspectrum. 


\subsubsection{Homogeneous medium}

An isotropic elastic homogeneous medium in three dimensions can be viewed as an open homogeneous waveguide of unbounded section in the $(x, y)$ plane ( $z$ being the axial direction). Introducing a PML of finite thickness in both directions $x$ and $y$, the problem becomes closed. A boundary condition, defined later, must be applied at the PML ends. Let us denote $\ell_{x}$ and $\ell_{y}$ the half thicknesses of the whole cross-section $\left(\ell_{x}=d_{x}+h_{x}\right.$, $\left.\ell_{y}=d_{y}+h_{y}\right) . \tilde{\ell}_{x}$ and $\tilde{\ell}_{y}$ are the complex half thicknesses, defined as follows:

$$
\tilde{\ell}_{x}=\int_{0}^{\ell_{x}} \gamma_{x}(\xi) \mathrm{d} \xi=d_{x}+\hat{\gamma}_{x} h_{x}, \quad \tilde{\ell}_{y}=\int_{0}^{\ell_{y}} \gamma_{y}(\xi) \mathrm{d} \xi=d_{y}+\hat{\gamma}_{y} h_{y} .
$$

Analytical solution. Applying the Helmholtz decomposition [45], the displacement vector $\tilde{\boldsymbol{u}}$ is written as $\tilde{\boldsymbol{u}}=$ $\tilde{\nabla} \tilde{\phi}+\tilde{\nabla} \wedge \tilde{\psi}$ (with $\tilde{\nabla} \cdot \tilde{\psi}=0$ ), where $\tilde{\phi}$ and $\tilde{\psi}=\left[\begin{array}{lll}\tilde{\psi}_{x} & \tilde{\psi}_{y} & \tilde{\psi}_{z}\end{array}\right]^{T}$ are scalar and vector potentials corresponding to longitudinal $(l)$ and shear $(s)$ waves respectively. The equilibrium equations of elastodynamics yield the uncoupled differential equations for potentials:

$$
\frac{\partial^{2} \tilde{\phi}}{\partial \tilde{x}^{2}}+\frac{\partial^{2} \tilde{\phi}}{\partial \tilde{y}^{2}}+\left(\frac{\omega^{2}}{c_{l}^{2}}+\lambda\right) \tilde{\phi}=0 \quad \text { and } \quad \frac{\partial^{2} \tilde{\psi}}{\partial \tilde{x}^{2}}+\frac{\partial^{2} \tilde{\psi}}{\partial \tilde{y}^{2}}+\left(\frac{\omega^{2}}{c_{s}^{2}}+\lambda\right) \tilde{\psi}=0
$$

where $\lambda=-k^{2} . c_{l}$ and $c_{s}$ are the bulk velocities of longitudinal and shear waves respectively.

Free or Dirichlet boundary conditions couple the potentials $\tilde{\phi}$ and $\tilde{\psi}$ so that fully analytical solutions are not achievable. Instead, the following mixed boundary conditions are considered:

$$
\tilde{u}_{x}=0, \quad \tilde{\sigma}_{x y}=0, \quad \tilde{\sigma}_{x z}=0 \text { at } \tilde{x}= \pm \tilde{\ell}_{x} \text { and } \tilde{u}_{y}=0, \quad \tilde{\sigma}_{x y}=0, \quad \tilde{\sigma}_{y z}=0 \text { at } \tilde{y}= \pm \tilde{\ell}_{y} .
$$

It can be shown that such boundary conditions yield uncoupled boundary conditions for $\tilde{\phi}$ and $\tilde{\psi}$ :

$$
\left\{\begin{array} { l } 
{ \frac { \partial \tilde { \phi } } { \partial \tilde { x } } = 0 \text { at } \tilde { x } = \pm \tilde { \ell } _ { x } } \\
{ \frac { \partial \tilde { \phi } } { \partial \tilde { y } } = 0 \text { at } \tilde { y } = \pm \tilde { \ell } _ { y } }
\end{array} \quad \left\{\begin{array} { l } 
{ \frac { \partial \tilde { \psi } _ { x } } { \partial \tilde { x } } = 0 \text { at } \tilde { x } = \pm \tilde { \ell } _ { x } } \\
{ \tilde { \psi } _ { x } = 0 \text { at } \tilde { y } = \pm \tilde { \ell } _ { y } }
\end{array} \quad \left\{\begin{array} { l } 
{ \tilde { \psi } _ { y } = 0 \text { at } \tilde { x } = \pm \tilde { \ell } _ { x } } \\
{ \frac { \partial \tilde { \psi } _ { y } } { \partial \tilde { y } } = 0 \text { at } \tilde { y } = \pm \tilde { \ell } _ { y } }
\end{array} \quad \left\{\begin{array}{l}
\tilde{\psi}_{z}=0 \text { at } \tilde{x}= \pm \tilde{\ell}_{x} \\
\tilde{\psi}_{z}=0 \text { at } \tilde{y}= \pm \tilde{\ell}_{y}
\end{array}\right.\right.\right.\right. \text {. }
$$

By separating the variables $\tilde{x}$ and $\tilde{y}$, the eigenvalues of the problem (17) and (19) are given by

$$
\lambda_{l}^{(p, q)}=-\frac{\omega^{2}}{c_{l}^{2}}+\left(\frac{p \pi}{2 \tilde{\ell}_{x}}\right)^{2}+\left(\frac{q \pi}{2 \tilde{\ell}_{y}}\right)^{2}, \lambda_{s}^{(m, n)}=-\frac{\omega^{2}}{c_{s}^{2}}+\left(\frac{m \pi}{2 \tilde{\ell}_{x}}\right)^{2}+\left(\frac{n \pi}{2 \tilde{\ell}_{y}}\right)^{2}
$$

where $m, n, p, q$ are integers. These apparently formal calculations can be easily justified following the same ideas as in [33].

From Eq. (20), it can be seen that two spectra occur instead of one with scalar waveguides [30, 43]. These two spectra correspond to compressional and shear waves respectively.

Without PML, the eigenvalues are real $\left(\tilde{\ell}_{x}=\ell_{x}, \tilde{\ell}_{y}=\ell_{y}\right)$. In the initial unbounded problem, $\ell_{x}$ and $\ell_{y}$ tend to infinity: the spectra in terms of $\lambda=-k^{2}$ tend to two real continuous half-lines $\left[-\omega^{2} / c_{l}^{2},+\infty\left[\right.\right.$ and $\left[-\omega^{2} / c_{s}^{2},+\infty[\right.$. These continua of eigenmodes are the so-called radiation modes, which are standing waves oscillating in the transverse directions. As simply shown by Eq. (20), each continuum is discretized by the truncation of crosssection at some finite distance.

With PML, the eigenvalues $\lambda$ are no longer real. The associated modes are still referred to as radiation modes since they are oscillating inside the PML. In the complex $\lambda$-plane, the eigenvalues of Eq. (20) belong to two angular sectors of origins $-\omega^{2} / c_{l}^{2}$ and $-\omega^{2} / c_{s}^{2}$. Each sector is limited by two half-lines of rotation angles $-2 \arg \tilde{\ell}_{x}$ and $-2 \arg \tilde{\ell}_{y}$. For clarity, these half-lines are denoted as

$$
\left(\Delta_{x}^{l / s}\right):-\omega^{2} / c_{l / s}^{2}+\mathbb{R}^{+} / \tilde{\ell}_{x}^{2}, \quad\left(\Delta_{y}^{l / s}\right):-\omega^{2} / c_{l / s}^{2}+\mathbb{R}^{+} / \tilde{\ell}_{y}^{2} .
$$

Each sector reduces to half-lines if $\arg \tilde{\ell}_{x}=\arg \tilde{\ell}_{y}$. When $\left|\tilde{\ell}_{x}\right|$ and $\left|\tilde{\ell}_{y}\right|$ increase, each spectrum of radiation modes gets denser and becomes continuous when $\left|\tilde{\ell}_{x}\right|$ and $\left|\tilde{\ell}_{y}\right|$ tend to $\infty$. 


\begin{tabular}{lccccc}
\hline \hline Material & $c_{l}(\mathrm{~m} / \mathrm{s})$ & $c_{s}(\mathrm{~m} / \mathrm{s})$ & $\rho\left(\mathrm{kg} / \mathrm{m}^{3}\right)$ & $\beta_{l}(\mathrm{~Np} /$ wavelength $)$ & $\beta_{s}(\mathrm{~Np} /$ wavelength $)$ \\
\hline Steel & 5960 & 3260 & 7932 & 0.003 & 0.008 \\
Concrete & 4222.1 & 2637.5 & 2300 & 0.0 & 0.0 \\
Stiff stone & 5720 & 3300 & 2200 & 0.0 & 0.0 \\
Grout & 2810 & 1700 & 1600 & 0.043 & 0.100 \\
\hline \hline
\end{tabular}

Table 1: Material characteristics.

From Eq. (16), the rotation angles of $\left(\Delta_{x}^{l / s}\right)$ and $\left(\Delta_{y}^{l / s}\right)$ are respectively

$$
-2 \arg \tilde{\ell}_{x}=-2 \arg \left(d_{x}+\hat{\gamma}_{x} h_{x}\right), \quad-2 \arg \tilde{\ell}_{y}=-2 \arg \left(d_{y}+\hat{\gamma}_{y} h_{y}\right) .
$$

This shows that each angle does not depend on the PML function profile itself (only its average value has an effect). It is noteworthy that the result given by Eq. (22) agrees with that obtained in Ref. [46] from a mathematical study of the scalar acoustic PML problem.

Numerical example. A concrete medium is considered. The material characteristics are given in Tab. 1. The PML interface positions are chosen as $d_{x}=d_{y}=d$ and the PML thicknesses are set to $h_{x}=h_{y}=3 d$. The whole section is a square of half-thicknesses $\ell_{x}=\ell_{y}=4 d$.

Figure 2 shows the dimensionless spectrum $\lambda d^{2}$ at the dimensionless angular frequency $\Omega=\omega d / c_{s}=1$ with different values of $\hat{\gamma}_{x}$ and $\hat{\gamma}_{y}$.

As shown in Figs. 2a and 2c, the analytical eigenvalues given by Eq. (20) belong to two angular sectors. They are limited by two half-lines $\left(\Delta_{x}^{l / s}\right)$ and $\left(\Delta_{y}^{l / s}\right)$ defined in Eq. (21), rotated from the real axis by angles of $-74^{\circ}$ and $-113^{\circ}$ respectively, in agreement with Eq. (22). As can be seen from Figs. $2 b$ and $2 d$, the angular sectors are reduced to half-lines when PML parameters are identical in both directions.

Numerical eigenvalues computed by the SAFE-CPML method are also shown in Fig. 2 (crosses). The finite elements are six-node triangles, whose average length is denoted by $l_{e}$. The PML functions $\gamma_{x}$ and $\gamma_{y}$ are parabolic, as defined by Eq. (15).

Numerical results are in agreement with the analytical ones, except for poles that are far from the real axis. Such poles indeed correspond to higher order modes (higher values of $p, q, m, n$ in Eq. (20)), which have high transverse wavenumbers, i.e. small transverse wavelengths. As in conventional eigenvalue FE problems, these modes are not well approximation due to the FE discretization. Refining the mesh allows to improve numerical results, as confirmed by Figs. 2c and 2d.

\subsubsection{Embedded cylindrical elastic waveguides}

In this subsection, numerical experiments are conducted on a cylindrical waveguide embedded into a softer solid matrix (the bulk velocities of the core are greater than in the surrounding medium). The test case is taken from the paper of Castaings et al. [24]. It consists of a steel cylinder of $10 \mathrm{~mm}$ radius buried in concrete. The material characteristics are given in Tab. 1. The steel is considered as elastic in this test $\left(\beta_{l}=\beta_{s}=0\right.$, where $\beta_{l}$ and $\beta_{s}$ denote the longitudinal and shear bulk wave attenuations in Neper per wavelength).

Contrary to the previous subsection, the eigenspectrum now includes leaky modes in addition to radiation modes (note that no trapped modes occur in this test case).

Numerical parameters. The radius of the circular core section is denoted by $a$. A Dirichlet condition is applied at the exterior boundary of the truncated section. As shown in Fig. 3, finite elements are six-node triangles. PML functions are parabolic. The PML parameters in the $x$ and $y$ directions are identical: $d_{x}=d_{y}, h_{x}=h_{y}, \hat{\gamma}_{x}=\hat{\gamma}_{y}$. The PML thicknesses are equal to $0.9 a$.

Numerical eigenspectrum. Figure 4 represents the dimensionless numerical spectrum $\lambda a^{2}$ for various PML parameters at the dimensionless frequency $\Omega=\omega a / c_{s_{0}}=3.86$ (where $c_{s_{0}}$ is the shear wave velocity of the core). Two kinds of modes can be distinguished. 


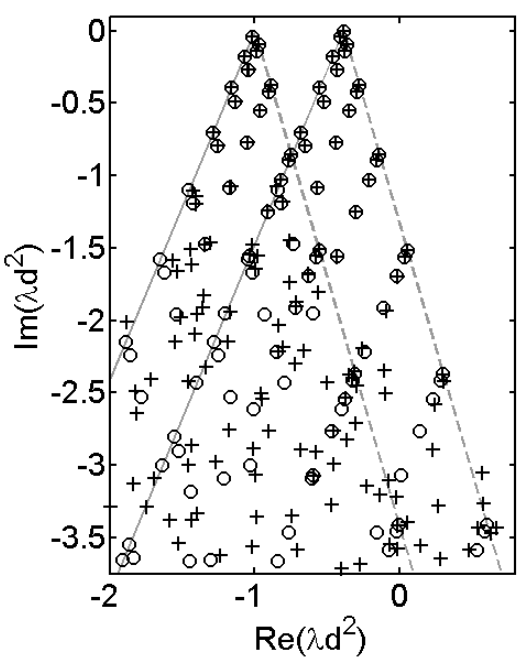

(a)

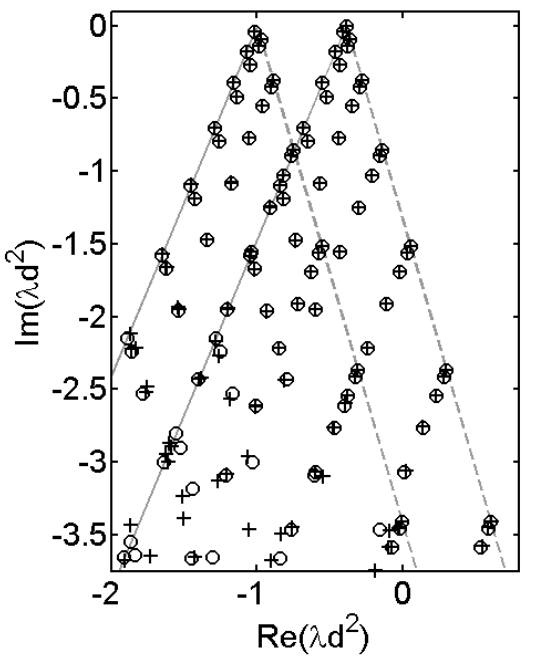

(c)

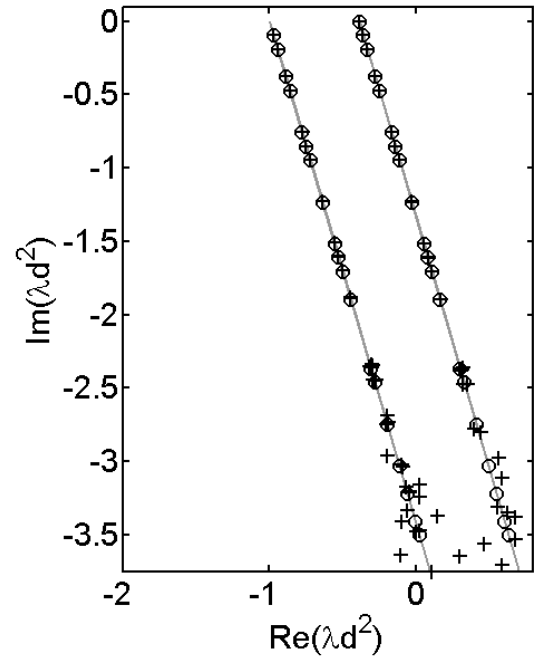

(b)

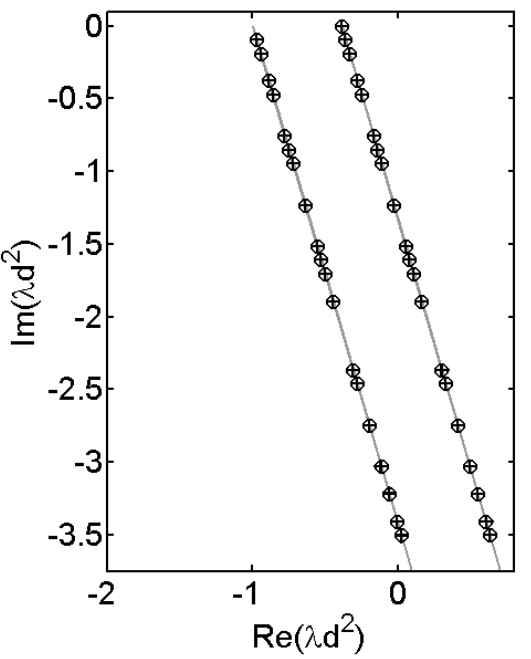

(d)

Figure 2: Spectrum of homogeneous concrete medium with section truncated by Cartesian PML at $\Omega=1\left(d_{x}=d_{y}=d, h_{x}=h_{y}=3 d\right)$ for: (a) $\hat{\gamma}_{x}=1+\mathrm{i}, \hat{\gamma}_{y}=1+2 \mathrm{i}, l_{e}=0.4 d$, (b) $\hat{\gamma}_{x}=\hat{\gamma}_{y}=1+\mathrm{i}, l_{e}=0.4 d$, (c) $\hat{\gamma}_{x}=1+\mathrm{i}, \hat{\gamma}_{y}=1+2 \mathrm{i}, l_{e}=0.2 d$ and (d) $\hat{\gamma}_{x}=\hat{\gamma}_{y}=1+\mathrm{i}, l_{e}=0.2 d$. Crosses: SAFE-CPML results, circles: analytical results. Dashed lines: $\left(\Delta_{x}^{l / s}\right)$, continuous lines: $\left(\Delta_{y}^{l / s}\right)$. 


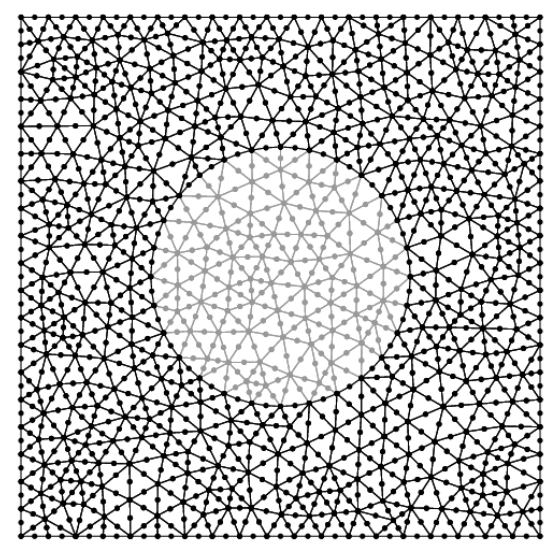

Figure 3: Cross-section mesh of an embedded cylindrical bar using Cartesian PML $\left(l_{e}=0.2 a\right)$.

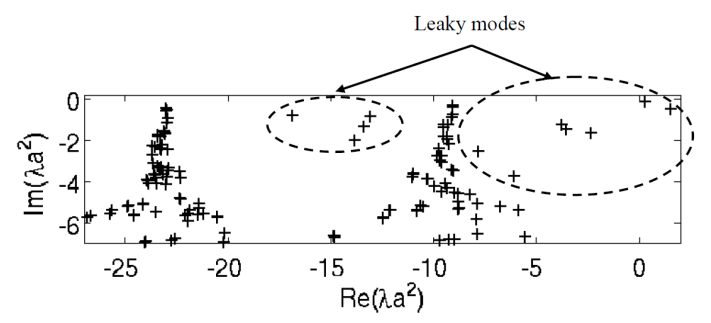

(a)

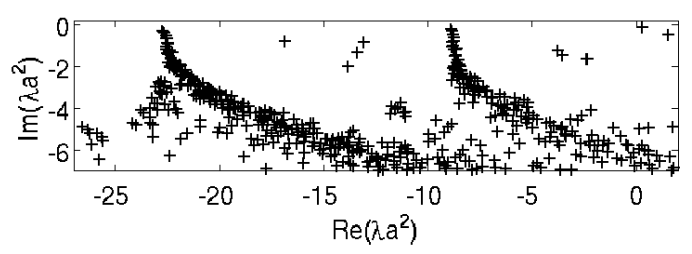

(c)

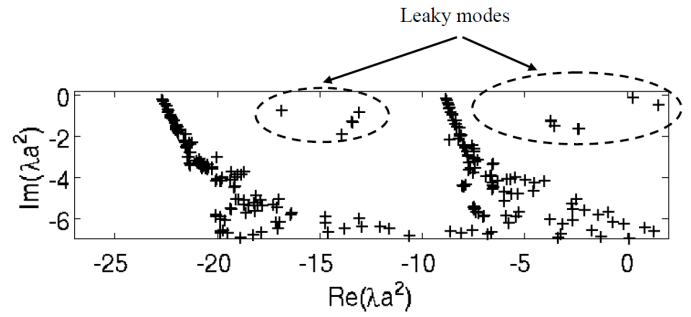

(b)

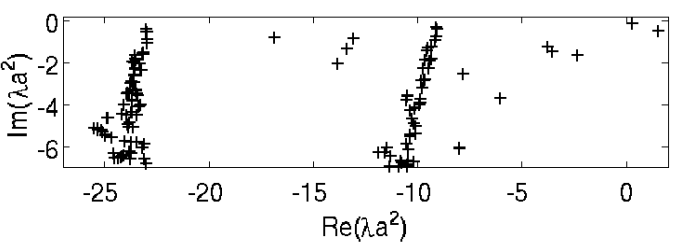

(d)

Figure 4: Numerical spectrum of steel-concrete waveguide obtained at $\Omega=\omega a / c_{s_{0}}=3.86$ by the SAFE-CPML method $\left(h_{x}=h_{y}=0.9 a\right)$ for: (a) $\hat{\gamma}_{x}=\hat{\gamma}_{y}=2+4 \mathrm{i}, d_{x}=d_{y}=1.1 a, l_{e}=0.2 a$, (b) $\hat{\gamma}_{x}=\hat{\gamma}_{y}=5+4 \mathrm{i}, d_{x}=d_{y}=1.1 a, l_{e}=0.2 a$, (c) $\hat{\gamma}_{x}=\hat{\gamma}_{y}=2+4 \mathrm{i}, d_{x}=d_{y}=3 a, l_{e}=0.2 a$, (d) $\hat{\gamma}_{x}=\hat{\gamma}_{y}=2+4 \mathrm{i}, d_{x}=d_{y}=1.1 a, l_{e}=0.1 a$ 
The first kind corresponds to radiation modes, yielding two spectra of origins $\left(-\omega^{2} / c_{l}^{2}, 0\right)$ and $\left(-\omega^{2} / c_{s}^{2}, 0\right)$, where $c_{l}$ and $c_{s}$ denote the bulk longitudinal and shear wave velocities of the embedding medium (concrete). Similarly to Sec. 2.5.1, these spectra correspond to the discretized continua of longitudinal and shear waves. Modes near the origins approximately form straight lines rotated from the real axis with angles equal to $107^{\circ}$ in Fig. $4 \mathrm{a}$ and $61^{\circ}$ in Fig. 4b. These angles are in agreement with the analytical formula given by Eq. (22). Far from their origins, radiation modes deviate from straight lines. Such modes are high order modes which the FE mesh can no longer approximate. This is confirmed by Fig. 4d, obtained with a refined mesh (the deviation occurs at a greater distance from the origins).

The rotation of radiation modes indeed allows to discover a second kind of modes, hidden in the original problem without PML. These modes are leaky modes. As observed in Fig. 4, the number of discovered leaky modes grows as the rotation angle is increased: compared to Fig. $4 \mathrm{a}\left(\hat{\gamma}_{x}=\hat{\gamma}_{y}=2+4 \mathrm{i}\right)$, two leaky modes are not present in Fig. $4 \mathrm{~b}\left(\hat{\gamma}_{x}=\hat{\gamma}_{y}=4+4 \mathrm{i}\right)$. As expected from Sec. 2.4 , more leaky modes can be found by increasing the argument of $\hat{\gamma}_{x}$ and $\hat{\gamma}_{y}$.

The rotation angles can also be modified by adjusting the PML interface position $d_{x}$ and $d_{y}$. Comparing Fig. $4 \mathrm{c}$ with $4 \mathrm{a}$ shows that high values of $d_{x}$ and $d_{y}$ reduce the rotation angles, in agreement with Eq. (22). In Fig. 4c, note that two leaky modes are spoiled by the deviation of high order radiation modes.

In practice for computing leaky modes, the PML interface should be set close to the core as suggested in Refs. [33, 30, 35]. From a physical point of view, a PML interface too far from the core allows leaky modes to significantly grow before entering the PML, which can deteriorate their computation. This has been mathematically justified by the increase of the norm of the resolvent of the eigenproblem [30, 35].

The spectra of radiation modes can be densified by choosing higher values of PML parameters $\left(d_{x}, d_{y}\right),\left(h_{x}, h_{y}\right)$ or $\left(\hat{\gamma}_{x}, \hat{\gamma}_{y}\right)$, which increases the complex half-thicknesses $\left|\tilde{\ell}_{x}\right|$ and $\left|\tilde{\ell}_{y}\right|$ as explained in Sec. 2.5.1. As an example, compared to Figs. 4a, these spectra get denser in Figs. 4b and 4c, for which the parameters $\operatorname{Re} \hat{\gamma}_{x}=\operatorname{Re} \hat{\gamma}_{y}$ and $d_{x}=d_{y}$ have been increased respectively.

\section{SAFE - RPML method}

In this section, the SAFE-RPML formulation is introduced. Following the same approach as in Sec. 2, the associated eigenspectrum is briefly studied through analytical and numerical experiments.

\subsection{Combining SAFE and radial PML techniques}

The formulation (1) is rewritten in cylindrical coordinates as follows:

$$
\int_{\tilde{\Omega}} \delta \tilde{\boldsymbol{\epsilon}}^{T} \tilde{\boldsymbol{\sigma}} \tilde{r} \mathrm{~d} \tilde{r} \mathrm{~d} \theta \mathrm{d} z-\omega^{2} \int_{\tilde{\Omega}} \tilde{\rho} \delta \tilde{\mathbf{u}}^{T} \tilde{\mathbf{u}} \tilde{r} \mathrm{~d} \tilde{r} \mathrm{~d} \theta \mathrm{d} z=0
$$

where $\tilde{x}=\tilde{r} \cos \theta, \tilde{y}=\tilde{r} \sin \theta$. The tilde notation represents the introduction of a PML along the radial direction. Note that in the above formulation, vectors and tensors are written in cylindrical coordinates but still expressed in the Cartesian basis. In cylindrical coordinates, the operator $\mathbf{L}_{\tilde{S}}$ of the strain-displacement relation (2) is

$$
\mathbf{L}_{\tilde{S}}=\mathbf{L}_{x}\left(\cos \theta \frac{\partial}{\partial \tilde{r}}-\frac{\sin \theta}{\tilde{r}} \frac{\partial}{\partial \theta}\right)+\mathbf{L}_{y}\left(\sin \theta \frac{\partial}{\partial \tilde{r}}+\frac{\cos \theta}{\tilde{r}} \frac{\partial}{\partial \theta}\right) .
$$

Applying the PML technique in the radial direction, the formulation (23) can be interpreted as the analytical continuation of the equilibrium equations into the complex radial coordinate $\tilde{r}$, with

$$
\tilde{r}(r)=\int_{0}^{r} \gamma(\xi) \mathrm{d} \xi
$$

where $\gamma(r)$ is a complex function satisfying

- $\gamma(r)=1$ for $r \leq d$

- $\operatorname{Im}\{\gamma\}>0$ for $r>d$. 


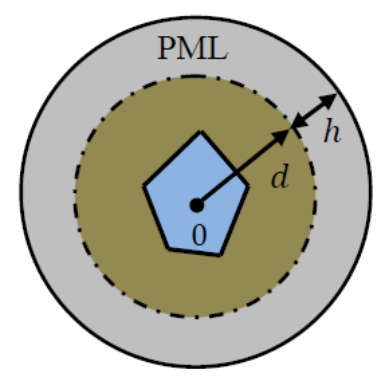

Figure 5: Open waveguide with cross-section truncated by radial PML.

$d$ is the position of the PML interface. As shown in Fig. 5, the cross-section of a SAFE-RPML problem is typically a circle of radius $d+h, h$ denoting the PML thickness. Similarly to Cartesian PML, the boundary condition applied at the PML exterior boundary can be arbitrarily chosen.

From Eq. (25), the change of variable $\tilde{r} \mapsto r$ yields for any function $\tilde{f}$ :

$$
\frac{\partial \tilde{f}}{\partial \tilde{r}}=\frac{1}{\gamma} \frac{\partial f}{\partial r}, \mathrm{~d} \tilde{r}=\gamma \mathrm{d} r
$$

where $\tilde{f}(\tilde{r}, \theta, z)=f(r, \theta, z)$. Applying this change of variable and the SAFE method to Eqs. (24) leads to an expression identical to Eq. (8), with

$$
\mathbf{L}_{S}=\mathbf{L}_{x}\left(\frac{\cos \theta}{\gamma} \frac{\partial}{\partial r}-\frac{\sin \theta}{\tilde{r}} \frac{\partial}{\partial \theta}\right)+\mathbf{L}_{y}\left(\frac{\sin \theta}{\gamma} \frac{\partial}{\partial r}+\frac{\cos \theta}{\tilde{r}} \frac{\partial}{\partial \theta}\right) .
$$

Before FE discretization, the formulation must be transformed back to Cartesian coordinates. The operator $\mathbf{L}_{S}$ then becomes

$$
\mathbf{L}_{S}=\mathbf{L}_{x}\left[\left(\frac{x^{2}}{\gamma r^{2}}+\frac{y^{2}}{\tilde{r} r}\right) \frac{\partial}{\partial x}+\left(\frac{1}{\gamma r^{2}}-\frac{1}{\tilde{r} r}\right) x y \frac{\partial}{\partial y}\right]+\mathbf{L}_{y}\left[\left(\frac{1}{\gamma r^{2}}-\frac{1}{\tilde{r} r}\right) x y \frac{\partial}{\partial x}+\left(\frac{y^{2}}{\gamma r^{2}}+\frac{x^{2}}{\tilde{r} r}\right) \frac{\partial}{\partial y}\right] .
$$

Finally, the FE discretization of the formulation along the cross-section yields the same form of eigenproblem as Eq. (9), but with the following elementary matrices:

$$
\begin{aligned}
\mathbf{K}_{1}^{e} & =\int_{e} \mathbf{N}^{e T} \mathbf{L}_{S}^{T} \mathbf{C} \mathbf{L}_{S} \mathbf{N}^{e} \frac{\tilde{r} \gamma}{r} \mathrm{~d} x \mathrm{~d} y, \mathbf{K}_{2}^{e}=\int_{e} \mathbf{N}^{e T} \mathbf{L}_{S}^{T} \mathbf{C} \mathbf{L}_{z} \mathbf{N}^{e} \frac{\tilde{r} \gamma}{r} \mathrm{~d} x \mathrm{~d} y \\
\mathbf{K}_{3}^{e} & =\int_{e} \mathbf{N}^{e T} \mathbf{L}_{z}^{T} \mathbf{C} \mathbf{L}_{z} \mathbf{N}^{e} \frac{\tilde{r} \gamma}{r} \mathrm{~d} x \mathrm{~d} y, \mathbf{M}^{e}=\int_{e} \rho \mathbf{N}^{e T} \mathbf{N}^{e} \frac{\tilde{r} \gamma}{r} \mathrm{~d} x \mathrm{~d} y .
\end{aligned}
$$

\subsection{PML absorbing function}

As suggested for Cartesian PML, a parabolic profile independent of frequency is chosen for the radial PML function $\gamma$, expressed as follows:

$$
\gamma(r)=\left\{\begin{array}{lll}
1 & \text { if } & r \leq d \\
1+3(\hat{\gamma}-1)\left(\frac{r-d}{h}\right)^{2} & \text { if } & r>d
\end{array}\right.
$$

where $\hat{\gamma}$ is the average value of $\gamma$ in the radial PML region:

$$
\hat{\gamma}=\frac{1}{h} \int_{d}^{d+h} \gamma(\xi) \mathrm{d} \xi
$$

For radial PML, the influence of $\hat{\gamma}$ on wave absorption can be illustrated by considering wave solutions in cylindrical coordinates. In the PML region $(r>d)$, the wave fields can be expressed by a combination of Hankel 
functions. Assuming negligible refection from the PML exterior boundary $\left(r=\ell_{r}=d+h\right)$, the radial dependence of wave fields is written as $H_{n}^{(1)}\left(k_{l / s} \tilde{r}\right)$, where $H_{n}^{(1)}$ is the Hankel function of first kind and $k_{l / s}$ denotes the radial wavenumber (shear or longitudinal).

Let us denote the complex radius $\tilde{\ell}_{r}$ by

$$
\tilde{\ell}_{r}=\int_{0}^{\ell_{r}} \gamma(\xi) \mathrm{d} \xi .
$$

For simplicity, we assume that the radial wavelength is small enough compared to $d$ and $\left|\tilde{\ell}_{r}\right|$ (i.e. $\left|k_{l / s} d\right| \gg 1$ and $\left.\left|k_{l / s} \tilde{\ell}_{r}\right| \gg 1\right)$. Then, the wave solutions at the PML interface and at the PML end, written in terms of $H_{n}^{(1)}\left(k_{l / s} d\right)$ and $H_{n}^{(1)}\left(k_{l / s} \tilde{\ell}_{r}\right)$ respectively, asymptotically behave like $\mathrm{e}^{\mathrm{i} k_{l / s} d} / \sqrt{k_{l / s} d}$ and $\mathrm{e}^{\mathrm{i} k_{l / s} \tilde{\ell}_{r}} / \sqrt{k_{l / s} \tilde{\ell}_{r}}=\left(\mathrm{e}^{\mathrm{i} k_{l / s} d} / \sqrt{k_{l / s} \tilde{\ell}_{r}}\right) \mathrm{e}^{\mathrm{i} k_{l / s} \hat{\gamma} h}$ respectively. Therefore, the total attenuation from the interface to the PML end can then be approximated by

$$
\frac{\left|H_{n}^{(1)}\left(k_{l / s} \tilde{\ell}_{r}\right)\right|}{\left|H_{n}^{(1)}\left(k_{l / s} d\right)\right|} \simeq \frac{\exp \left(-\left|k_{l / s}\right||\hat{\gamma}| h \sin \left(\arg k_{l / s}+\arg \hat{\gamma}\right)\right)}{\sqrt{\left|1+\frac{\hat{\gamma} h}{d}\right|}} .
$$

Concerning the numerator (exponential term), it can be noticed that the radial wavenumber $k_{l / s}$ plays the same role as in Eq. (12). Therefore, the influence of $\hat{\gamma}$ is similar to the effect of $\hat{\gamma}_{x}$ and $\hat{\gamma}_{y}$ with the Cartesian PML method, already described in Sec. 2.4.

The slight difference with radial PML is that this exponential term is modulated by an attenuation factor given by $1 / \sqrt{|1+\hat{\gamma} h / d|}$. Without PML $(\hat{\gamma}=1)$, this attenuation factor corresponds to the geometrical attenuation of cylindrical waves and is equal to $1 / \sqrt{1+h / d}$. A radial PML enhances this attenuation factor as $|\hat{\gamma}|$ increases.

\subsection{Eigenspectrum (homogeneous medium)}

The SAFE-RPML eigenspectrum is now briefly analyzed in order to understand how a radial PML acts on the eigenspectrum. The analytical solution of a homogeneous medium is derived. Compared to Cartesian coordinates, it is difficult with cylindrical coordinates to find appropriate boundary conditions leading to fully analytical solutions of the elastic problem. Therefore, the analytical solution of this section is obtained for the scalar wave equation of acoustics. The elastic problem will be handled through numerical experiments. For conciseness, this section is limited to the case of a homogeneous medium. The reader is referred to Appendix A for the analysis of a cylindrical core waveguide embedded into an infinite medium, where similarly to the homogeneous case, the analytical solution for an acoustic waveguide is first studied and numerical experiments in the elastic case are then performed.

A homogeneous medium can be considered as an open homogeneous waveguide of unbounded cross-section. Introducing a radial PML of finite thickness $h$ and of position $d$, the cross-section becomes bounded and of radius $\ell_{r}=d+h$.

Analytical solution for a scalar problem. The acoustic wave equation written in cylindrical coordinates is

$$
\frac{\mathrm{d}^{2} \tilde{\phi}}{\mathrm{d} \tilde{r}^{2}}+\frac{1}{\tilde{r}} \frac{\mathrm{d} \tilde{\phi}}{\mathrm{d} \tilde{r}}-\frac{n^{2}}{\tilde{r}^{2}} \tilde{\phi}+\left(\frac{\omega^{2}}{c^{2}}-k^{2}\right) \tilde{\phi}=0
$$

where $\tilde{\phi}$ denotes the acoustic variable, $n$ is the circumferential order and $c$ is the acoustic wave velocity.

The solution of Eq. (33) is $\tilde{\phi}(\tilde{r})=A J_{n}\left(k_{r} \tilde{r}\right)+B Y_{n}\left(k_{r} \tilde{r}\right)$, where $k_{r}$ is the radial wavenumber satisfying the relation $k_{r}^{2}+k^{2}=\omega^{2} / c^{2} . J_{n}$ and $Y_{n}$ are Bessel functions of the first kind and of the second kind respectively. Since $Y_{n}\left(k_{r} \tilde{r}\right)$ tends to infinity when $|\tilde{r}|$ tends to $0, B$ must vanish. A Dirichlet condition $\tilde{\phi}\left(\tilde{\ell}_{r}\right)=0$ is applied at the PML exterior boundary, yielding the characteristic equation: $J_{n}\left(k_{r} \tilde{\ell}_{r}\right)=0$. The eigenvalues are hence

$$
\lambda_{n m}=-k_{n m}^{2}=-\frac{\omega^{2}}{c^{2}}+\left(\frac{\chi_{n m}}{\tilde{\ell}_{r}}\right)^{2}
$$

where $\chi_{n m}$ denotes the $m$ th zero of $J_{n}(x)$. 


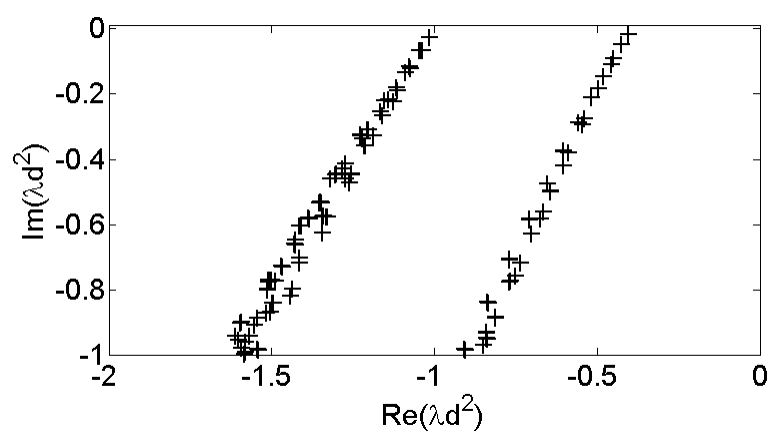

Figure 6: Dimensionless spectrum of homogeneous concrete medium computed with the SAFE-RPML method at $\omega d / c_{s}=1$. PML parameters are $h=3 d, \hat{\gamma}=2+4 \mathrm{i}\left(l_{e}=0.2 d\right)$.

Without PML $\left(\tilde{\ell}_{r}=\ell_{r}\right)$, the eigenvalues are real. In the initial problem of infinite section, $R$ tends to infinity and the $\lambda$ spectrum becomes a real continuous half-line $\left[-\omega^{2} / c^{2},+\infty[\right.$. This continuum can be referred to as the essential spectrum of radiation modes [30, 35].

With a radial PML, the eigenvalues $\lambda$ of radiation modes become complex. In the complex plane, they belong to a discretized half-line of rotation angle

$$
-2 \arg \tilde{\ell}_{r}=-2 \arg (d+\hat{\gamma} h)
$$

This coincides with the result obtained in Ref. [35] for the computation of acoustic resonances with a radial PML method. Similarly to the Cartesian PML (see Sec. 2.5.1), the discretized half-line gets denser when $\left|\tilde{\ell}_{r}\right|$ increases and the rotation angle is independent of the radial PML function profile.

Numerical solution for an elastic problem. The radiation modes of a homogeneous elastic problem are now computed by the SAFE-RPML method. A concrete medium is considered. Finite elements are six-node triangles, whose average length $l_{e}$ is chosen as $0.1 d$. The PML function $\gamma$ is parabolic, as defined by Eq. (29). The PML thickness is set to $h=3 d$. The radius of the whole cross-section is $\ell_{r}=4 d$.

Figure 6 shows the spectrum at the dimensionless frequency $\Omega=\omega d / c_{s}=1$ with $\hat{\gamma}=2+4 \mathrm{i}$. Instead of one half-line with the scalar problem, the eigenvalues belong to two discretized half-lines starting from $-\omega^{2} / c_{l}^{2}$ and $-\omega^{2} / c_{s}^{2}$. As already found in Sec. 2.5.1, these two spectra of radiation modes correspond to longitudinal and shear waves respectively (their deviation from straight lines being due to the poor FE approximation of higher order modes).

The half-lines are rotated from the real axis with equal rotation angles, approximately equal to $118^{\circ}$. This angle is in agreement with the acoustic formula (35).

\section{Dispersion curves}

In this section, the dispersion curves of leaky and trapped modes are computed by both the SAFE-CPML and the SAFE-RPML methods for three test cases taken from the literature. The calculation of modal properties in open waveguides is presented first (kinetic energy, energy velocity and group velocity).

\subsection{Kinetic energy}

By analytical continuation into complex coordinates, the cross-section and time averaged kinetic energy can be defined as $\bar{E}_{k}=\frac{1}{2} \int_{\tilde{S}} \tilde{\rho} \overline{\mathbf{v}} \cdot \tilde{\mathbf{v}} \mathrm{d} \tilde{S}$, where $\tilde{\mathbf{v}}=\mathrm{d} \tilde{\mathbf{u}} / \mathrm{d} t$ is the velocity vector and bars denote time averaging over one period. As already mentioned in Sec. 2.2, $\tilde{S}$ denotes the waveguide cross-section including the truncated PML.

This definition is rewritten by using the change of variables from complex to real coordinates, as

$$
\bar{E}_{k}=\frac{1}{4} \int_{S} \rho \operatorname{Re}\left(\mathbf{v}^{*} \cdot \mathbf{v}\right) \mathrm{d} \tilde{S}=\frac{\omega^{2}}{4} \int_{S} \rho \operatorname{Re}\left(\mathbf{u}^{*} \cdot \mathbf{u}\right) \mathrm{d} \tilde{S}=\frac{\omega^{2}}{4} \int_{S} \rho \mathbf{u}^{*} \cdot \mathbf{u} \mathrm{d} \tilde{S}
$$


where $\mathrm{d} \tilde{S}=\gamma_{x} \gamma_{y} \mathrm{~d} x \mathrm{~d} y$ for the SAFE-CPML method and $\mathrm{d} \tilde{S}=\tilde{r} \gamma / r \mathrm{~d} x \mathrm{~d} y$ for the SAFE-RPML method. The superscript $*$ refers to the complex conjugate transpose.

The FE expression of kinetic energy is given by

$$
\bar{E}_{k}=\frac{\omega^{2}}{4} \sum_{e} \int_{S^{e}} \rho \mathbf{u}^{e *} \cdot \mathbf{u}^{e} \mathrm{~d} \tilde{S}=\frac{\omega^{2}}{4} \sum_{e} \mathbf{U}^{e *}\left(\int_{S^{e}} \rho \mathbf{N}^{e T} \mathbf{N}^{e} \mathrm{~d} \tilde{S}\right) \mathbf{U}^{e}=\frac{\omega^{2}}{4} \mathbf{U}^{*} \mathbf{M U}
$$

This result is the same as for closed waveguides (without PML) [21, 47]. In the presence of PML, the slight difference is that $\mathbf{M}$ is no longer real and the kinetic energy becomes complex.

\subsection{Energy velocity}

For closed waveguides, the energy velocity is defined as follows [45]:

$$
v_{e}=\frac{\operatorname{Re}\left(\int_{S} \overline{\mathbf{P}} \cdot \mathbf{n d} S\right)}{\operatorname{Re}\left(\bar{E}_{k}\right)+\operatorname{Re}\left(\bar{E}_{p}\right)}
$$

where $\mathbf{P}$ is the complex Poynting vector given by $P_{j}=-\frac{1}{2} v_{i}^{*} \sigma_{i j} . \bar{E}_{p}$ denotes the cross-section and time averaged potential energy, given by $\bar{E}_{p}=\frac{1}{2} \int_{S} \overline{\boldsymbol{\epsilon}^{*} \boldsymbol{\sigma}} \mathrm{d} S . \mathbf{n}$ is the unit vector normal to the cross section (in the $z$ direction). The potential energy $\bar{E}_{p}$ and the complex power flow $\int_{S} \overline{\mathbf{P}} \cdot \mathbf{n d} S$ can be post-processed from SAFE matrices thanks to the following formula [47], here modified for complex wavenumbers:

$$
\int_{S} \overline{\mathbf{P}} \cdot \mathbf{n} d s=-\frac{\mathrm{i} \omega}{2} \mathbf{U}_{c}^{*}\left(\mathbf{K}_{2}+\mathrm{i} k \mathbf{K}_{3}\right) \mathbf{U}, \bar{E}_{k}=\frac{\omega^{2}}{4} \mathbf{U}^{*} \mathbf{M U}, \bar{E}_{p}=\frac{1}{4} \mathbf{U}^{*}\left(\mathbf{K}_{1}+\mathrm{i} k \mathbf{K}_{2}-\mathrm{i} k^{*} \mathbf{K}_{2}^{T}+k^{*} k \mathbf{K}_{3}\right) \mathbf{U} .
$$

For open waveguides, the definition of $v_{e}$ is similar to Eq. (38). However the integrals on the cross-section are usually restricted to the core region $[48,49]$, which avoids the transverse growth of leaky modes at infinity. Hence, Eqs. (38) and (39) still apply for open waveguides but $S$ must be replaced with $S_{0}$, which denotes the cross-section of the core.

\subsection{Group velocity}

The group velocity is usually defined by $v_{g}=\partial \omega / \partial k$. The calculation of $v_{g}$ can be achieved from the derivative of the SAFE eigensystem with respect to $k$, as done in Refs. [21, 50]. However, the definition $v_{g}=\partial \omega / \partial k$ only applies when the axial wavenumber $k$ is real. In open waveguides, the axial wavenumber of leaky modes is complex. The proper definition of group velocity for damped modes is $[48,51]$

$$
v_{g}=\frac{\mathrm{d} \omega}{\mathrm{dRe}(k)}=\left[\operatorname{Re}\left(\frac{\mathrm{d} k}{\mathrm{~d} \omega}\right)\right]^{-1} .
$$

Let $\left(k_{j}, \mathbf{U}_{j}^{+}\right)$denote an eigensolution of Eq. (9):

$$
\left(\mathbf{K}_{1}-\omega^{2} \mathbf{M}+\mathrm{i} k_{j}\left(\mathbf{K}_{2}-\mathbf{K}_{2}^{T}\right)+k_{j}^{2} \mathbf{K}_{3}\right) \mathbf{U}_{j}^{+}=\mathbf{0} .
$$

Taking the derivative of Eq. (41) with respect to $\omega$ yields

$$
\left(-2 \omega \mathbf{M}+\mathrm{i} \frac{\mathrm{d} k_{j}}{\mathrm{~d} \omega}\left(\mathbf{K}_{2}-\mathbf{K}_{2}^{T}\right)+2 k_{j} \frac{\mathrm{d} k_{j}}{\mathrm{~d} \omega} \mathbf{K}_{3}\right) \mathbf{U}_{j}^{+}=-\left(\mathbf{K}_{1}-\omega^{2} \mathbf{M}+\mathrm{i} k_{j}\left(\mathbf{K}_{2}-\mathbf{K}_{2}^{T}\right)+k_{j}^{2} \mathbf{K}_{3}\right) \frac{\mathrm{d} \mathbf{U}_{j}^{+}}{\mathrm{d} \omega} .
$$

As mentioned in Sec. 2.2, $\left(-k_{j}, \mathbf{U}_{j}^{-}\right)$is also a solution of Eq. (9). Left-multiplying Eq. (42) by the transpose of $\mathbf{U}_{j}^{-}$allows the right-hand term to vanish, which leads to the group velocity of the $j^{\text {th }}$ mode:

$$
v_{g_{j}}=\left[\operatorname{Re}\left\{\frac{2 \omega \mathbf{U}_{j}^{-T} \mathbf{M} \mathbf{U}_{j}^{+}}{\mathbf{U}_{j}^{-T}\left(i\left(\mathbf{K}_{2}-\mathbf{K}_{2}^{T}\right)+2 k_{j} \mathbf{K}_{3}\right) \mathbf{U}_{j}^{+}}\right\}\right]^{-1} .
$$

The calculation of $v_{g}$ for a given mode hence requires its opposite-going counterpart.

As opposed to the energy velocity, it should be noticed that the group velocity may generally be not appropriate for attenuated waves [48] (including leaky waves). Yet, the group velocity still applies for trapped modes, which are non-attenuated (in non-dissipative media). 


\subsection{Modal filtering}

According to Secs. 2.5, 3.3 and Appendix A, the SAFE-PML methods provide many radiation modes in addition to the modes of interest (leaky or trapped). Since radiation modes mainly oscillate inside the PML, they are dependent on the choice of PML parameters and cannot be considered as intrinsic to the physics. A large number of radiation modes perturbs the visualization of dispersion curves of trapped and leaky modes. A modal filtering must be processed to identify and remove these radiation modes. The filtering criterion proposed in this paper is based on the ratio of kinetic energy in the PML region over the kinetic energy of the whole cross-section. Physical modes are then identified if this criterion is smaller than a user-defined value $\eta_{\max }$ :

$$
\frac{\left|\bar{E}_{k_{P M L}}\right|}{\left|\bar{E}_{k}\right|}<\eta_{\max }
$$

where $\bar{E}_{k_{P M L}}$ is defined from Eq. (36) by replacing $S$ with $S_{P M L}$, which denotes the cross-section restricted to the PML region. Note that the modulus must be used in Eq. (44) since the kinetic energy is complex in the presence of PML. Note that $\eta_{\max }$ is independent of frequency in this paper. For a given FE mesh, the appropriate value of $\eta_{\max }$ essentially depends on the PML parameters, which have been assumed as constant with frequency (see Secs. 2.4 and 3.2).

\subsection{Results}

Three examples of open waveguides are considered. The first one has already been described in Secs. 2.5.2 and Appendix A (steel cylinder in concrete). The second test case is taken from the work of Pavlakovic [19] and is a $1 \mathrm{~mm}$ radius steel cylinder in stiff stone. This case is of interest due to the existence of one trapped mode. The third example, taken from Ref. [26], is a steel square bar buried in a viscoelastic grout, for which no analytical solution is available. Material characteristics are given in Tab. 1. The steel is considered as elastic for the first two examples $\left(\beta_{l}=\beta_{s}=0\right)$ and as viscoelastic in the last one $\left(\beta_{l}=0.003, \beta_{s}=0.043\right)$.

The PML position and thickness are set to $h_{x}=h_{y}=h=0.9 a$ and $d_{x}=d_{y}=d=1.1 a$, where $a$ denotes the radius of the cylindrical core for the first two test cases and the half-thickness of the squared core for the third case. Note that with the SAFE-RPML formulation, the PML could be set to $d=a$ when the core is cylindrical. A Dirichlet condition is applied at the PML exterior boundaries (numerical tests have shown that other boundary conditions yield small differences on trapped and leaky modes). The finite elements are six-node triangles whose length satisfies the following meshing criterion: $l_{e} \leq \lambda_{m} / 5$, where $\lambda_{m}=\min \left(c_{s}\right) / \max (f)\left(\min \left(c_{s}\right)\right.$ is the lowest shear velocity of the problem). The SAFE-PML dispersion curves are shown after modal filtering, the parameter $\eta_{\max }$ being specified for each example.

Let us consider the first test case. The FE meshes are shown in Figs. 3 and 7. According to the eigenspectrum analyses in Secs. 2.5.2 and Appendix A, the eigenvalues of leaky modes are located around $\left|\omega / c_{l}\right|$ in the complex plane. The computation has been centered around $+\omega / c_{l}$ in order to reduce the number of eigenvalues to solve. In this way, only positive-going modes are calculated. 300 modes have been computed at each frequency with the SAFE-CPML method, against 150 modes with the SAFE-RPML method. Actually, the SAFE-RPML method appears more advantageous from a computational point of view for this example because the core is of circular shape: the PML region can be smaller, which also decreases the number of radiation modes to solve. Besides, 5139 dofs (degrees of freedom) are generated with the SAFE-CPML method against 3975 dofs with the SAFERPML method.

In order to illustrate the efficiency of the filtering criterion proposed in Sec. 4.4, Fig. 8 shows the phase velocity dispersion curves obtained by both SAFE-PML methods without modal filtering. Clearly, the presence of many radiation modes in the dispersion curves prevents the identification of leaky modes.

Figure 9 represents the kinetic energy ratio of modes obtained by the SAFE-RPML method at $200 \mathrm{kHz}$. It can be observed that radiation modes yields a ratio approximatively equal to 1 because they mainly oscillate in the PML region, as opposed to leaky modes. Figure 9 shows that the energy kinetic ratio allows to well distinguish the radiation modes from the others and that an appropriate value of the threshold $\eta_{\max }$ is rather easy to choose (here, a threshold value of 0.75 turns out to be satisfying).

Figure 10 represents the dispersion curves with modal filtering. The curves of leaky modes are now clearly observed (compare Figs. 10a and 8). We point out that other energy ratio, based on Poynting vector or potential 


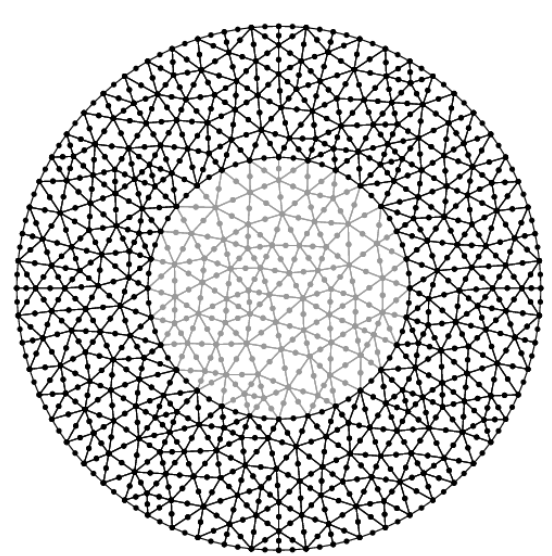

Figure 7: Cross-section mesh of an embedded cylindrical bar using radial PML $\left(l_{e}=0.2 a\right)$.

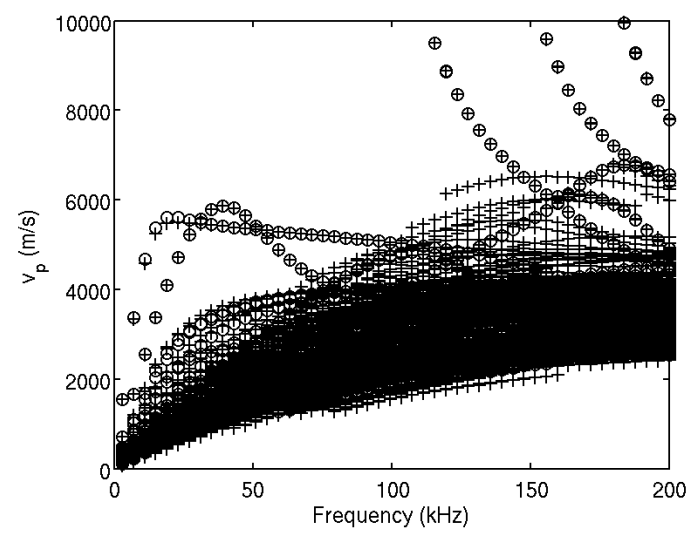

Figure 8: Phase velocity dispersion curves of steel-concrete cylindrical waveguide without filter $\left(\eta_{\max }=1\right)$. Crosses: SAFE-CPML results, circles: SAFE-RPML results. PML parameters are: $\hat{\gamma}_{x}=\hat{\gamma}_{y}=\hat{\gamma}=2+4 \mathrm{i}, d_{x}=d_{y}=d=1.1 a, h_{x}=h_{y}=h=0.9 a$.

energy, have been experimented. However, our numerical tests have not shown any improvement in the filtering of radiation modes (results not shown for paper conciseness). Therefore, the kinetic energy based ratio is preferred owing to its simpler post-processing.

The axial attenuation expressed in $\mathrm{dB} / \mathrm{m}$ (Fig. 10b) is defined by $\alpha=8.686 \mathrm{Im} k$. Note that for calculating the group velocity (Fig. 10d), it was also necessary to compute the negative-going modes by searching eigenvalues around $-\omega / c_{l}$.

The dispersion curves obtained with the SAFE-CPML and the SAFE-RPML methods are superimposed, which tends to show that both methods yield the same order of accuracy. The curves shown in Fig. 10a, b and c are in good agreement with the results obtained by the SAFE-AL technique [24] or the SAFE-BEM method [26]. This validates the SAFE-PML approaches proposed in this paper. Note that the AL thickness used in Ref. [24] is 16 times larger than the core radius, so approximately 16 times larger than the PML used in this paper, yielding dispersion curves that are not so accurate as those obtained by the PML method.

It can be observed that the group velocity curves differ quite significantly from the energy velocity (compare Fig. 10c and d). One recalls that Bernard et al. [48] have shown that this difference disappears for modes with no attenuation but always exists for attenuated modes (such as leaky modes).

Figure 11 shows the axial displacement field of a given mode, here the leaky compressional L $(0,1)$ mode, obtained with each method at $103.8 \mathrm{kHz}$. In the PML region, it can be observed that the CPML absorption strength is non-axially symmetric, as opposed to the RPML method. However, the modeshapes are found to be identical in the physical region (i.e. excluding the PML zone). 


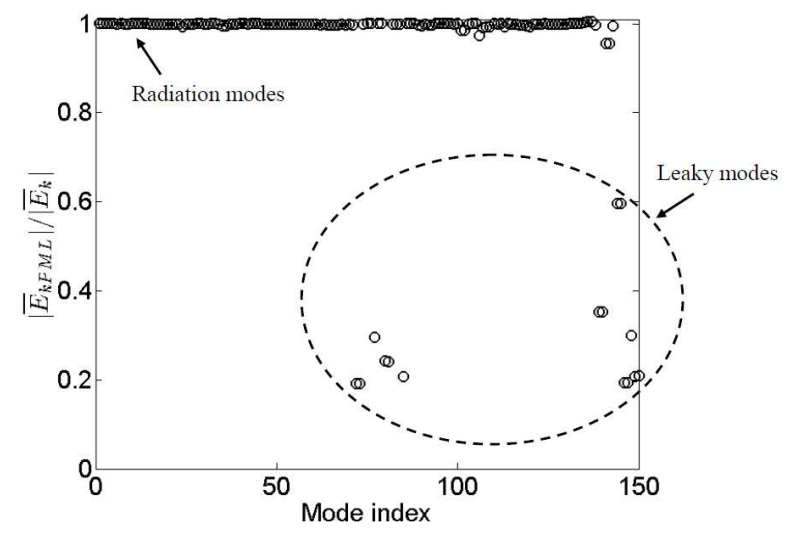

Figure 9: Kinetic energy ratio of modes in steel-concrete cylindrical waveguide obtained by the SAFE-RPML method at $200 \mathrm{kHz}$. PML parameters are: $\hat{\gamma}=2+4 \mathrm{i}, d=1.1 a, h=0.9 a$.

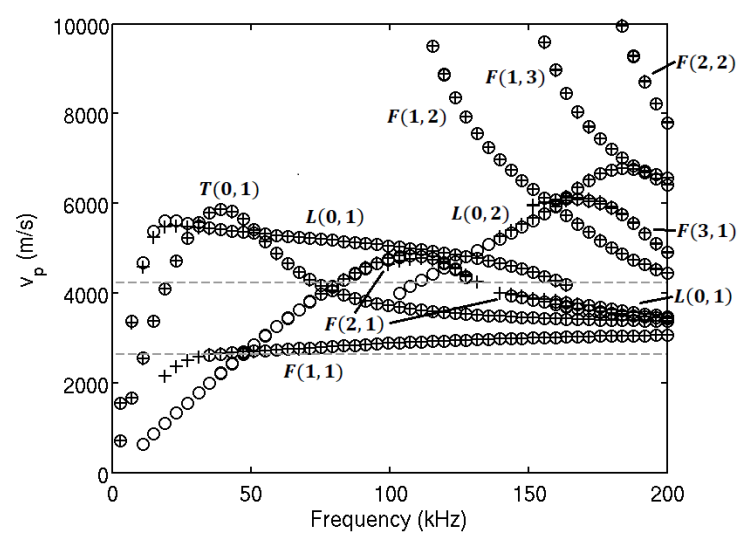

(a)

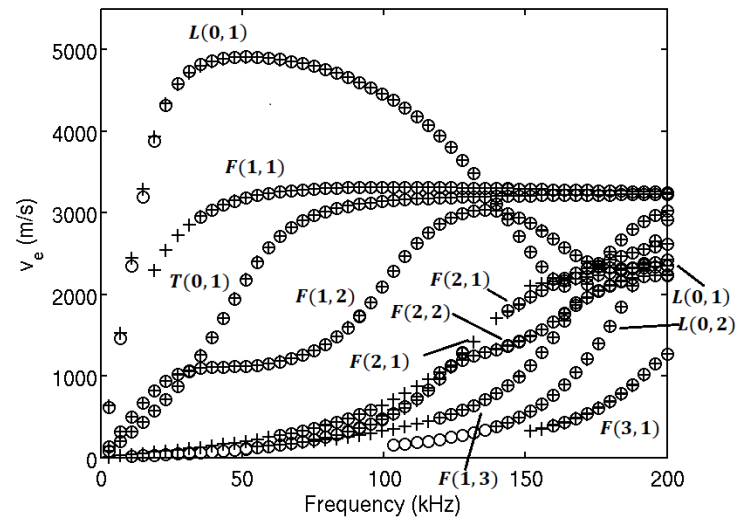

(c)

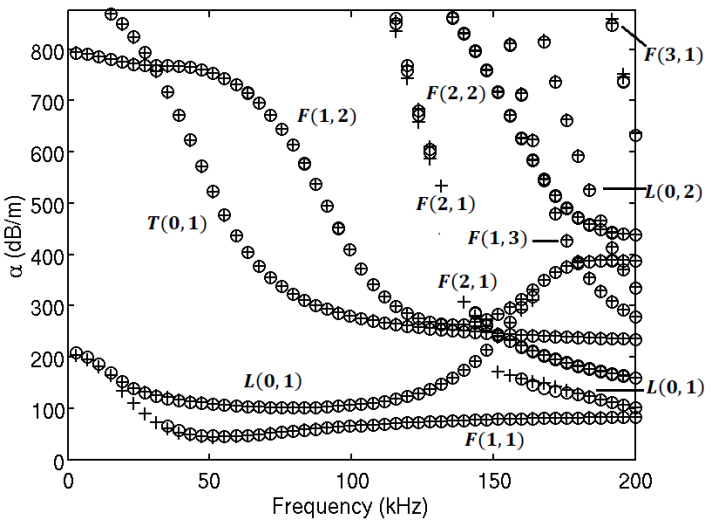

(b)

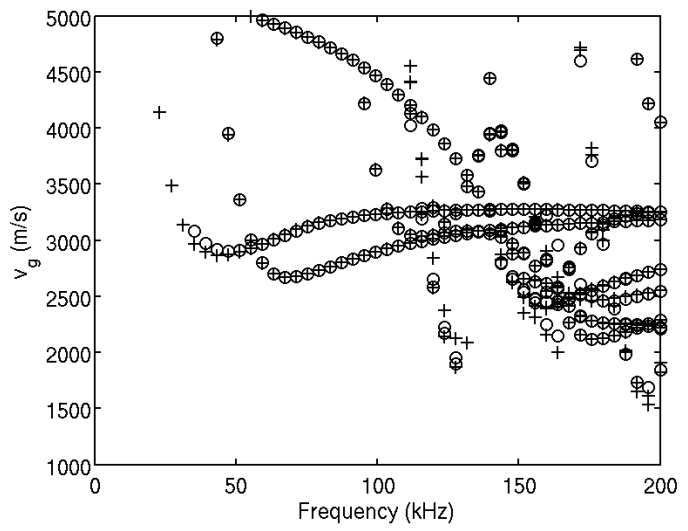

(d)

Figure 10: Dispersion curves of steel-concrete cylindrical waveguide for: (a) phase velocity, (b) axial attenuation, (c) energy velocity and (d) group velocity. Crosses: SAFE-CPML results $\left(\eta_{\max }=0.9\right)$, circles: SAFE-RPML results $\left(\eta_{\max }=0.75\right)$. PML parameters are: $\hat{\gamma}_{x}=\hat{\gamma}_{y}=\hat{\gamma}=$ $2+4 \mathrm{i}, d_{x}=d_{y}=d=1.1 a, h_{x}=h_{y}=h=0.9 a$. 


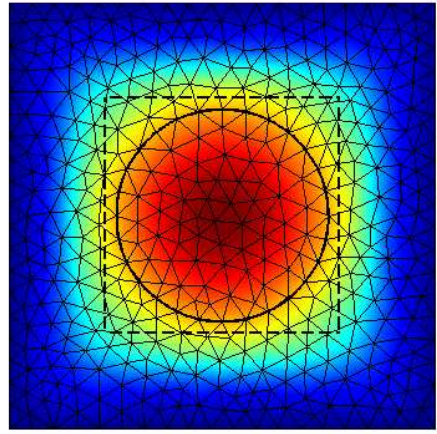

(a)

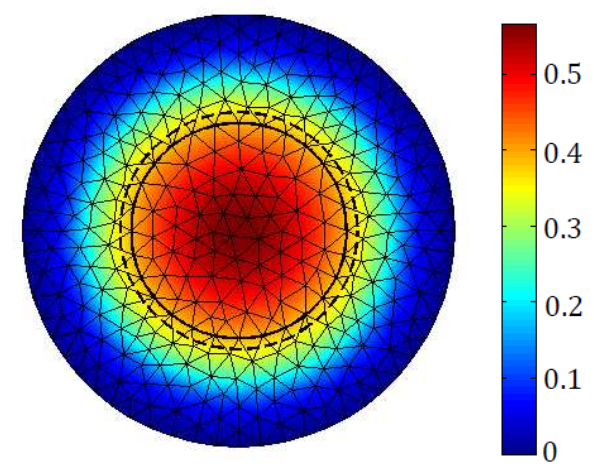

(b)

Figure 11: Modulus of axial displacement of the $\mathrm{L}(0,1)$ mode at $103.8 \mathrm{kHz}$ (steel-concrete cylindrical waveguide) obtained by (a) SAFECPML and (b) SAFE-RPML methods. Dashed line: PML interface, continuous line: material interface.

From a physical point of view, it can be noticed that the $\mathrm{L}(0,1)$ and $\mathrm{F}(2,1)$ curves are discontinuous around $163 \mathrm{kHz}$ and $135 \mathrm{kHz}$ respectively. These discontinuities are physical and occur when the phase velocity $v_{p}$ crosses the bulk wave velocities $c_{l}$ or $c_{s}$ of the surrounding medium (concrete) - see Fig. 10a for instance. For a given mode, the partial longitudinal (or shear) wave actually changes from leaky to trapped when $v_{p}$ becomes lower than $c_{l}$ (or $c_{s}$ ) [19].

In practice, the calculation of modes having a phase velocity at the vicinity of $c_{l}$ and $c_{s}$ can be difficult due to the undefined nature of partial waves (Fig. 10) $[19,24,26]$. This problem is also encountered with SAFE-PML methods because the transverse wavenumbers become small when $v_{p}$ is close to $c_{l}$ or $c_{s}$. According to Eqs. (12) and (32), large values of PML thickness or PML function would be required to sufficiently attenuate waves.

The same problem is also encountered for the computation of modes at low frequencies. For instance, in Fig. 10, leaky modes are still found to exist for phase velocities of a given mode smaller than the bulk shear velocity of the surrounding media, which does not seem physically correct. These low frequency modes are indeed not properly computed. Restricting the analysis to a low frequency range, one remedy would be to enhance the PML absorption strength by increasing the PML thickness or the average value of PML functions.

Figure 12 represents the phase velocity and the attenuation for the second test case (steel-stiff stone waveguide). The FE meshes used with Cartesian and radial PML methods are the same as in the first test case. In this example, the $\mathrm{F}(1,1)$ mode is trapped $(\operatorname{Im} k=0)$ while the other modes are leaky. In the initial problem without PML, trapped modes are located on the real axis of the complex plane. Their wavenumber in absolute value is larger than $\omega / c_{s}[4,52]$. Hence in this test case, the computation has been centered around $\omega / c_{s}$ instead of $\omega / c_{l}$. For each frequency, 265 and 180 modes have been solved for the SAFE-CPML and the SAFE-RPML methods respectively.

Since both trapped and leaky modes exist, the real and imaginary parts of $\hat{\gamma}_{x}, \hat{\gamma}_{y}$ and $\hat{\gamma}$ have been set to a sufficiently large value $\left(\hat{\gamma}_{x}=\hat{\gamma}_{y}=\hat{\gamma}=4+4 \mathrm{i}\right)$ in order to attenuate both kinds of modes. Results are in agreement with those of Ref. [19] calculated by an analytical approach. In particular, the trapped mode is well approximated by SAFE-PML methods (except for lowest frequencies, for the same reason as mentioned in the previous test case).

For the third test case (viscoelastic steel-grout squared waveguide), the SAFE-CPML method has been preferred because the cross-section of the core is of rectangular shape. The FE mesh is shown in Fig. 13, generating 5127 dofs. 600 modes have been solved, centered round $\omega / c_{l}$. Figure 14 shows the dispersion curves in phase velocity and attenuation. The modes have been labeled as in Ref. [26]. Here again, numerical results are in good agreement with literature results, obtained in Ref. [26] with a SAFE-BEM technique. Note that a poor approximation of lowest frequency modes possibly occurs for the same reasons as in the previous test case. 


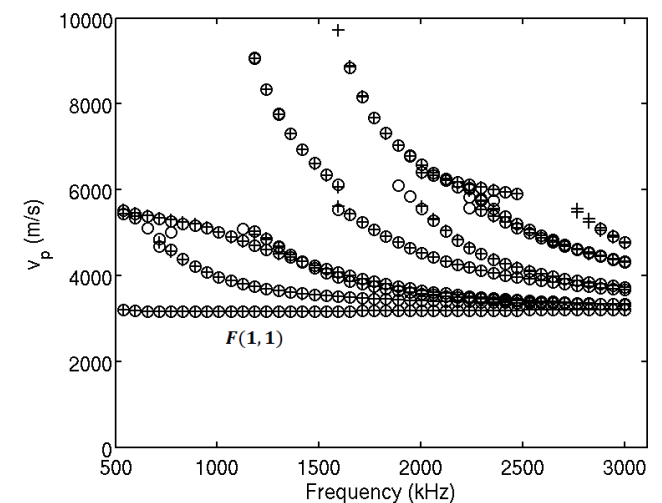

(a)

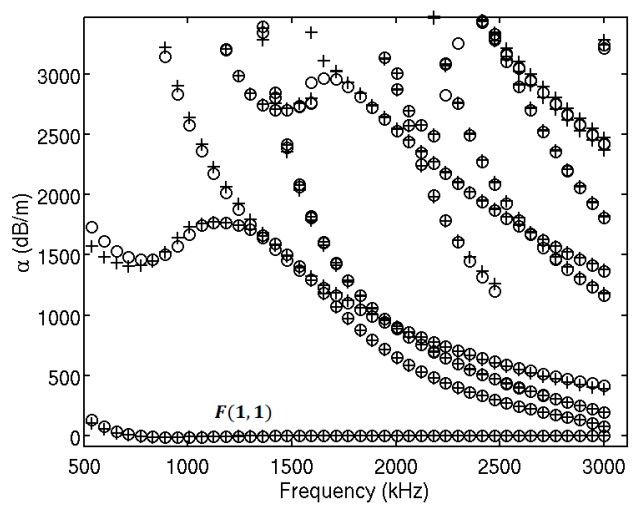

(b)

Figure 12: Dispersion curves of steel-stiff stone cylindrical waveguide for: (a) phase velocity and (b) axial attenuation. Crosses: SAFECPML results $\left(\eta_{\max }=0.75\right)$, circles: SAFE-RPML results $\left(\eta_{\max }=0.7\right)$. PML parameters are: $\hat{\gamma}_{x}=\hat{\gamma}_{y}=\hat{\gamma}=4+4 \mathrm{i}, d_{x}=d_{y}=d=1.1 a$, $h_{x}=h_{y}=h=0.9 a$.

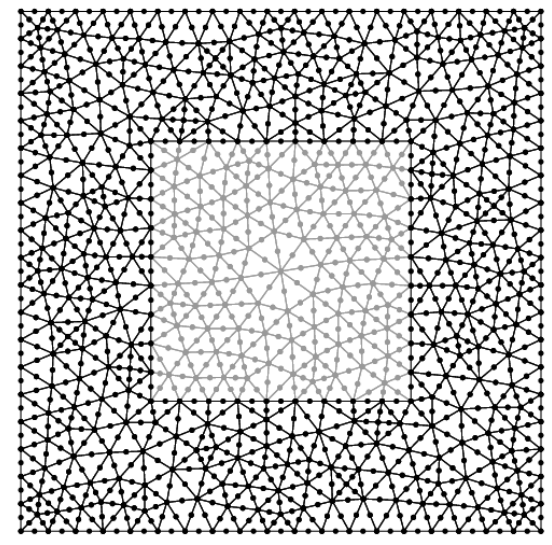

Figure 13: Cross-section mesh of an embedded square bar using Cartesian PML $\left(l_{e}=0.2 a\right)$.

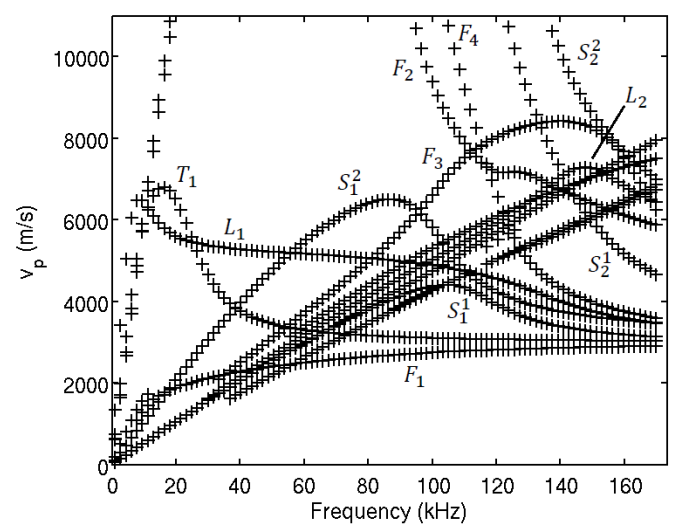

(a)

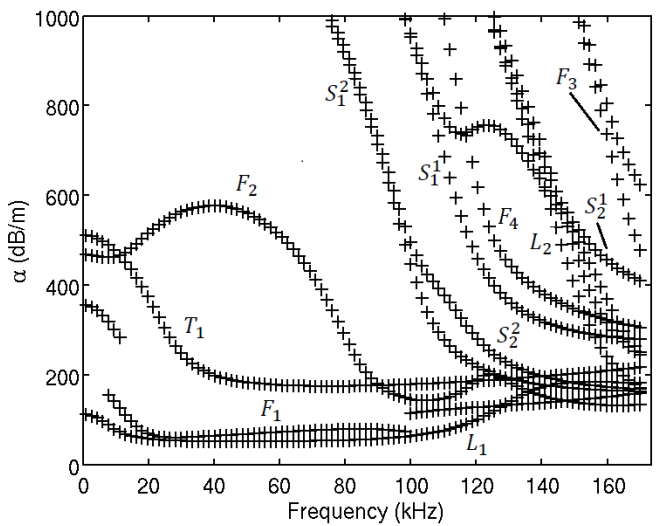

(b)

Figure 14: Dispersion curves of a viscoelastic rectangular steel bar buried in grout computed by the SAFE-CPML method for: (a) phase velocity and (b) axial attenuation. PML parameters are: $\hat{\gamma}_{x}=\hat{\gamma}_{y}=\hat{\gamma}=2+4 \mathrm{i}, d_{x}=d_{y}=d=1.1 a, h_{x}=h_{y}=h=0.9 a\left(\eta_{\max }=0.9\right)$. 


\section{Conclusion}

In this work, SAFE-PML methods have been applied to compute the eigenmodes of three-dimensional open elastic waveguides. Two kinds of PML, Cartesian and radial, have been implemented.

The spectral objects obtained from the proposed numerical approach have been clarified and the effects of PML parameters on radiation and leaky modes have been highlighted through analytical solutions and numerical experiments. The radiation modes of open elastic waveguides are shown to belong to two continua corresponding to longitudinal and shear waves respectively. The PML rotates these continua in the complex plane. The truncation of the PML discretizes them, with a densification by increasing the so-called complex thickness (or radius). The rotation angle of radiation modes are shown to be independent of PML function profiles and must be large enough to discover the leaky modes of interest.

For three test cases taken from the literature, the dispersion curves computed by both SAFE-CPML and SAFE-RPML methods provide satisfying results, for leaky modes as well as trapped modes. A modal filtering criterion has been proposed. This criterion is based on the ratio of kinetic energy in the PML region over the whole section and allows to efficiently remove the radiation modes in the visualization of dispersion curves. As far as the computational time is concerned, the SAFE-RPML appears to be more suitable for modeling open waveguides of circular cross-section. Conversely, the Cartesian PML should be preferred when the cross-section of the core is rectangular.

The main drawback of the approach is the presence of many radiation modes, which significantly increases the computational time. These modes are not intrinsic to the physics (since they depend on PML parameters) and are not of interest for NDE. Another drawback is the appropriate choice of PML parameters. Such a drawback is inherent to any problem involving PML and is also encountered with the AL technique. When the reference results are not known a priori, a convergence study is necessary. For instance for a given profile of PML function, the convergence of numerical results can be investigated by refining the finite element size and increasing the PML thickness. Note that the appropriate PML interface position is rather straightforward to choose as the PML should be set as close as possible to the core in order to limit the transverse growth of leaky waves.

\section{Appendix A. Eigenspectrum of embedded cylindrical waveguides with radial PML}

Analytical solution of scalar problem. In this analytical study, one considers a cylindrical acoustic waveguide of radius $a$ embedded into an infinite medium. A radial PML of finite thickness $h$ is introduced at the position $d \geq a$. The total radius of cross-section including PML is $\ell_{r}=d+h$.

The system of equations in radial complex coordinates is given by two Helmholtz equations (one for each medium). Continuity conditions are applied at the interface $r=a$ between both media and a Dirichlet condition is applied at the PML exterior boundary:

$$
\phi_{0}(a)=\tilde{\phi}(a), \frac{1}{\rho_{0}} \frac{\mathrm{d} \phi_{0}}{\mathrm{~d} r}(a)=\frac{1}{\rho} \frac{\mathrm{d} \tilde{\phi}}{\mathrm{d} \tilde{r}}(a), \quad \tilde{\phi}\left(\tilde{\ell}_{r}\right)=0 .
$$

$\phi_{0}$ and $\tilde{\phi}$ denote the acoustic variables in the core and in the surrounding medium respectively. $\rho_{0}$ and $\rho$ are the mass densities of the core and of the exterior medium. We assume that the core and the surrounding medium are homogeneous: fluid properties are constant (the tilde has been dropped for conciseness).

It can be readily shown that the dispersion relation associated to this problem is

$$
J_{n}^{\prime}\left(k_{r_{0}} a\right)\left[Y_{n}\left(k_{r} a\right) J_{n}\left(k_{r} \tilde{\ell}_{r}\right)-Y_{n}\left(k_{r} \tilde{\ell}_{r}\right) J_{n}\left(k_{r} a\right)\right] \frac{k_{r_{0}}}{\rho_{0}}-J_{n}\left(k_{r_{0}} a\right)\left[Y_{n}^{\prime}\left(k_{r} a\right) J_{n}\left(k_{r} \tilde{\ell}_{r}\right)-Y_{n}\left(k_{r} \tilde{\ell}_{r}\right) J_{n}^{\prime}\left(k_{r} a\right)\right] \frac{k_{r}}{\rho}=0
$$

where $k_{r_{0}}=\sqrt{\omega^{2} / c_{0}^{2}-k^{2}}$ and $k_{r}=\sqrt{\omega^{2} / c^{2}-k^{2}}$ are the transverse wavenumbers in each medium $\left(c_{0}\right.$ and $c$ denote the velocities of the core and of the exterior medium respectively).

The above equation cannot be solved analytically. Concerning radiation modes, further insight can be gained from an asymptotic point of view. Assuming a large complex radius $\left(\left|\tilde{\ell}_{r} / a\right| \gg 1\right)$, the transverse wavelength of low order radiation modes can be considered as sufficiently large to neglect the influence of the core: such modes 


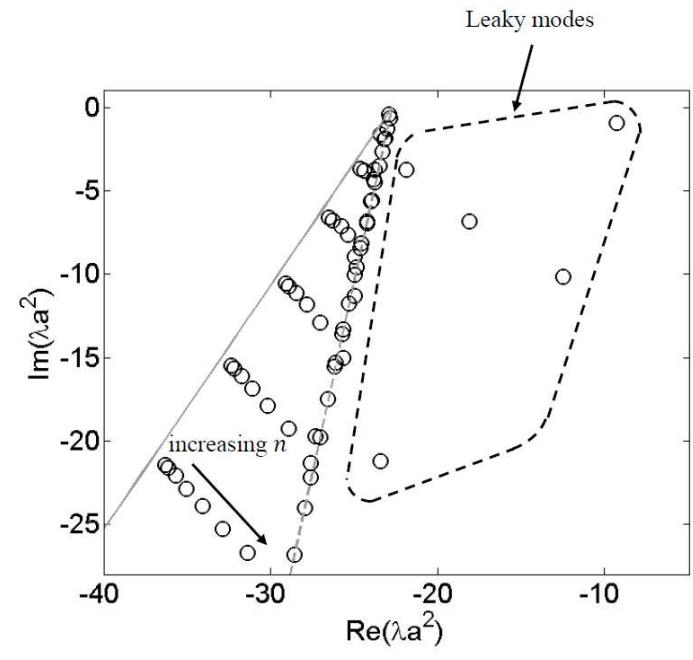

(a)

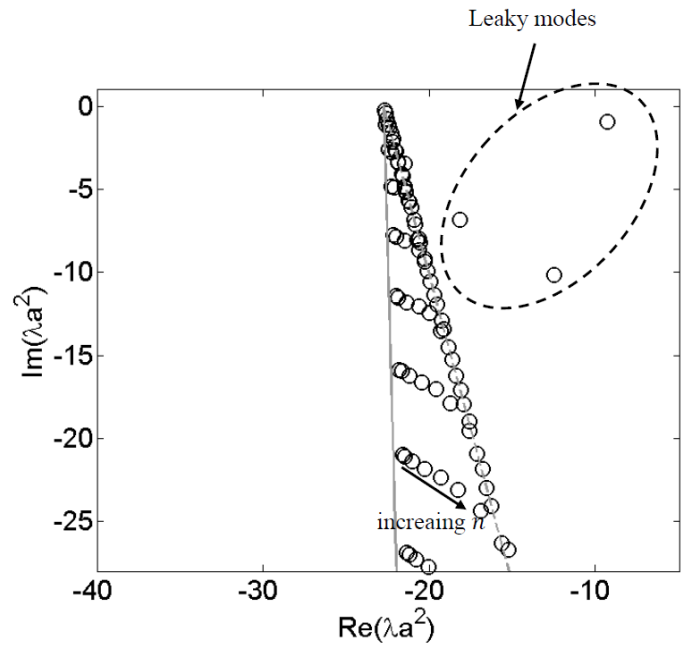

(b)

Figure A.15: Analytical spectrum of an embedded cylindrical acoustic waveguide at $\Omega=\omega a / c_{0}=3.86\left(\rho=0.29 \rho_{0}, c=0.81 c_{0}\right)$ with $d=1.1 a, h=0.9 a$ for: (a) $\hat{\gamma}=2+4 \mathrm{i}$ and (b) $\hat{\gamma}=4+4 \mathrm{i}$. Dashed line: $\left(\Delta_{1}\right)$, continuous line: $\left(\Delta_{2}\right)$.

almost satisfy the homogeneous medium equation derived in Sec. 3.3, i.e. $J_{n}\left(k_{r} \tilde{\ell}_{r}\right) \simeq 0$, yielding a first asymptote $\left(\Delta_{1}\right)$ :

$$
\left(\Delta_{1}\right):-\omega^{2} / c^{2}+\mathbb{R}^{+} / \tilde{\ell}_{r}^{2}
$$

which is a half-line of origin $-\omega^{2} / c^{2}$ rotated by $-2 \arg \tilde{\ell}_{r}$ in the complex plane.

Conversely, it can be shown that for a given $n$, the eigenvalues $\lambda$ of high order radiation modes (low transverse wavelength) tend toward a second asymptote $\left(\Delta_{2}\right)$ defined by

$$
\left(\Delta_{2}\right):-\omega^{2} / c^{2}+\mathbb{R}^{+} /\left(\tilde{\ell}_{r}-a\right)^{2}
$$

yielding a half-line of origin $-\omega^{2} / c^{2}$ rotated by $-2 \arg \left(\tilde{\ell}_{r}-a\right)$. Details are not shown for paper conciseness. Similar asymptotic developments can be found in Ref. [30].

Figure A.15 shows an example of eigensolutions of Eq. (A.2) for $d=1.1 a, h=0.9 a$ and different values of $\hat{\gamma}$. In this example, $\left(\rho_{0}, c_{0}\right)$ and $(\rho, c)$ are the mass densities and the shear velocities of steel and concrete respectively (see Tab. 1). The circumferential order $n$ varies from 0 to 10 . As expected, the eigenspectrum contains both leaky modes and radiation modes. It can be observed that the radiation modes appear to be included inside a sector delimited by $\left(\Delta_{1}\right)$ and $\left(\Delta_{2}\right)$. More precisely, radiation modes are found to be located on one branch asymptotic to $\left(\Delta_{1}\right)$ and sub-branches. In each sub-branch, the radiation modes get closer to $\left(\Delta_{1}\right)$ as the circumferential order $n$ increases.

Note that the rotation angles of $\left(\Delta_{1}\right)$ and $\left(\Delta_{2}\right)$ are also independent of the PML function profile $\gamma$ because one has

$$
-2 \arg \tilde{\ell}_{r}=-2 \arg (d+\hat{\gamma} h), \quad-2 \arg \left(\tilde{\ell}_{r}-a\right)=-2 \arg (d+\hat{\gamma} h-a) .
$$

Compared to Fig. A.15a, the angular sector of radiation modes is reduced in Fig. A.15b. This is due to the fact that increasing Re $\hat{\gamma}$ increases $\operatorname{Re} \tilde{\ell}_{r}$, which reduces the difference between the rotation angles of $\left(\Delta_{1}\right)$ and $\left(\Delta_{2}\right)$ (this can be readily shown from Eq. (A.5)). Given that leaky modes are discovered in the region where $\arg \lambda<-2 \arg \tilde{\ell}_{r}$, more leaky modes are found in Fig. A.15a than in Fig. A.15b thanks to a greater rotation angle of $\left(\Delta_{1}\right)$.

Without core $(a=0)$, note that Eqs. (A.3) and (A.4) yields equal rotation angles for $\left(\Delta_{1}\right)$ and $\left(\Delta_{2}\right)$ : the radiation modes sector is reduced to one discretized half-line rotated by $-2 \arg \tilde{\ell}_{r}$, which coincides with the result of Sec. 3.3. 


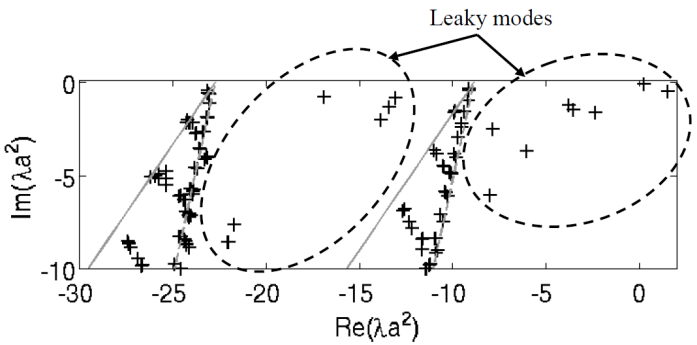

(a)

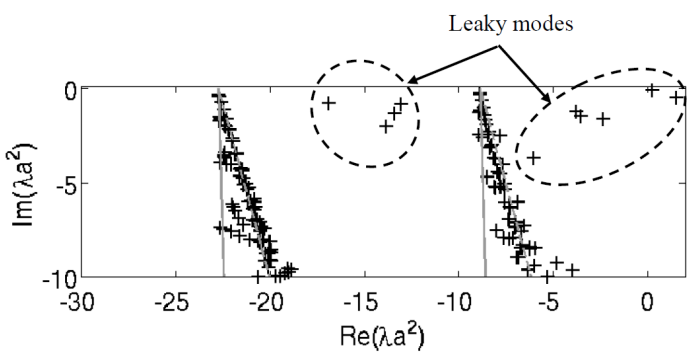

(b)

Figure A.16: Numerical spectrum of steel-concrete waveguide obtained by the SAFE-RPML method at $\Omega=3.86$ with $d=1.1 a, h=0.9 a$ $\left(l_{e}=0.1 a\right)$ for: (a) $\hat{\gamma}=2+4 \mathrm{i}$ and (b) $\hat{\gamma}=4+4 \mathrm{i}$. Dashed line: $\left(\Delta_{1}^{l / s}\right)$, continuous line: $\left(\Delta_{2}^{l / s}\right)$.

Numerical experiments on an elastic waveguide. The same test case as in Sec. 2.5.2 is considered (elastic steelconcrete waveguide). Figure 7 shows the example of a cross-section mesh used with the SAFE-RPML method $\left(l_{e}=0.2 a\right)$. Note that the effects of radial PML interface position and mesh size on eigenspectrum are identical to Cartesian PML (see Sec. 2.5.2). Therefore this subsection focuses on the effects of $\hat{\gamma}$ specific to the SAFE-RPML method.

Figure A.16 represents the eigenspectrum computed by the SAFE-RPML method at the dimensionless frequency $\Omega=3.86$. As suggested for Cartesian PML, the radial PML interface is set close to the core $(d=1.1 a)$. The average length of finite elements is $l_{e}=0.1 a$.

As shown for the scalar problem in the previous subsection, it can be noticed that the radiation elastic modes are included in sectors. Instead of one, the elastic problem yields two sectors, associated with longitudinal and shear waves respectively. Each sector is limited by two half-lines, denoted as $\left(\Delta_{1}^{l / s}\right)$ and $\left(\Delta_{2}^{l / s}\right)$, of rotation angles approximately equal to $100^{\circ}$ and $120^{\circ}$ in Fig. A.16a ( $73^{\circ}$ and $88^{\circ}$ in Fig. A.16b). These angles are closed to the acoustic formula (A.5). Similarly to the acoustic case, each sector is formed by one branch asymptotic to $\left(\Delta_{1}^{l / s}\right)$ and sub-branches and angular sectors are reduced as Re $\hat{\gamma}$ increases (compare Figs. A.16b with A.16a). Similarly to the SAFE-CPML method, increasing $|\hat{\gamma}|$ densifies the spectrum of radiation modes.

Concerning leaky modes, only those of arguments lower than the angle of $\left(\Delta_{1}^{l / s}\right)$ can be discovered (i.e. $\arg \lambda<-2 \arg \tilde{\ell}_{r}$ ), which explains why less leaky modes are found in Fig. A.16b than in Fig. A.16a.

To conclude this section, it is worth noting that the leaky modes shown in Fig. A.16 are in agreement with those of Fig. 4, obtained the SAFE-RPML method.

\section{Acknowledgements}

This work has been partially funded by the French Agence Nationale de la Recherche in frame of the Programme Inter Carnot Fraunhofer PICF of the Franco-German FilameNDT project.

\section{References}

[1] V. Maupin, The radiation modes of a vertically varying half-space: A new representation of the complete Green's function in terms of modes, Geophysical Journal International 126 (3) (1996) 762-780.

[2] L. Margerin, Generalized eigenfunctions of layered elastic media and application to diffuse fields, Journal of the Acoustical Society of America 125 (1) (2009) 164-174.

[3] R. E. Collin, Field Theory of Guided Waves, IEEE Press, 1991.

[4] R. N. Thurston, Elastic-waves in rods and clad rods, Journal of the Acoustical Society of America 64 (1) (1978) 1-37.

[5] A. Bamberger, Y. Dermenjian, P. Joly, Mathematical analysis of the propagation of elastic guided waves in heterogeneous media, Journal of Differential Equations 88 (1) (1990) 113-154.

[6] J. Hu, C. R. Menyuk, Understanding leaky modes: slab waveguide revisited, Advances in Optics and Photonics 1 (1) (2009) 58-106.

[7] J. A. Simmons, E. Drescher-Krasicka, H. N. G. Wadley, Leaky axisymmetric modes in infinite clad rods. 1, Journal of the Acoustical Society of America 92 (2) (1992) 1061-1090.

[8] L. E. Alsop, A. S. Goodman, S. Gregersen, Reflection and transmission of inhomogeneous waves with particular application to Rayleigh waves, Bulletin of the Seismological Society of America 64 (6) (1974) 1635-1652. 
[9] A. C. Hladky-Hennion, P. Langlet, M. de Billy, Conical radiating waves from immersed wedges, Journal of the Acoustical Society of America 108 (6) (2000) 3079-3083.

[10] T. Vogt, M. Lowe, P. Cawley, The scattering of guided waves in partly embedded cylindrical structures, Journal of the Acoustical Society of America 113 (3) (2003) 1258 - 1272.

[11] G. Cavigilia, A. Morro, Inhomogeneous Waves in Solids and Fluids, World Scientific Publishing Co. Pte. Ltd., Farrer Road, Singapore, 1992.

[12] C. H. Chapman, Lamb's problem and comments on the paper "On leaking modes" by Usha Gupta, Pure and Applied Geophysics 94 (1) (1972) $233-247$.

[13] P. D. Hislop, I. M. Sigal, Introduction to Spectral Theory. With Applications to Schrödinger Operators, Applied Mathematical Sciences 113, Springer-Verlag, New York, 1996.

[14] M. J. S. Lowe, Plate waves for the NDT of diffusion bonded titanium, Ph.D. thesis, Mechanical Engineering Department, Imperial College London (1992).

[15] M. D. Beard, M. J. S. Lowe, Non-destructive testing of rock bolts using guided ultrasonic waves, International Journal of Rock Mechanics and Mining Sciences 40 (4) (2003) 527-536.

[16] L. Laguerre, A. Grimault, M. Deschamps, Ultrasonic transient bounded-beam propagation in a solid cylinder waveguide embedded in a solid medium, Journal of the Acoustical Society of America 121 (4) (2007) 1924 - 1934.

[17] M. J. S. Lowe, P. Cawley, Comparison of the modal porperties of a stiff layer embedded in a solid medium with the minima of the plane-wave reflection coefficient, Journal of the Acoustical Society of America 97 (3) (1995) 1625-1637.

[18] M. J. S. Lowe, P. Cawley, The influence of the modal properties of a stiff layer embedded in a solid medium on the field generated in the layer by a finite-sized transducer, Journal of the Acoustical Society of America 97 (3) (1995) 1638-1649.

[19] B. N. Pavlakovic, Leaky guided ultrasonic waves in NDT, Ph.D. thesis, Mechanical Engineering Department, Imperial College London (1998).

[20] L. Gavric, Computation of propagative waves in free rail using a finite element technique, Journal of Sound and Vibration 185 (3) (1995) 531-543.

[21] I. Bartoli, A. Marzani, F. Lanza di Scalea, E. Viola, Modeling wave propagation in damped waveguides of arbitrary cross-section, Journal of Sound and Vibration 295 (2006) 685-707.

[22] T. Hayashi, W.-J. Song, J. L. Rose, Guided wave dispersion curves for a bar with an arbitrary cross-section, a rod and rail example, Ultrasonics 41 (3) (2003) 175-183.

[23] P. W. Loveday, Semi-analytical finite element analysis of elastic waveguides subjected to axial loads, Ultrasonics 49 (2009) $298-300$.

[24] M. Castaings, M. Lowe, Finite element model for waves guided along solid systems of arbitrary section coupled to infinite solid media, Journal of the Acoustical Society of America 123 (2) (2008) 696-708.

[25] Z. Fan, M. J. S. Lowe, M. Castaings, C. Bacon, Torsional waves propagation along a waveguide of arbitrary cross section immersed in a perfect fluid, Journal of the Acoustical Society of America 124 (4) (2008) 2002-2010.

[26] M. Mazzotti, I. Bartoli, A. Marzani, E. Viola, A coupled SAFE-2.5D BEM approach for the dispersion analysis of damped leaky guided waves in embedded waveguides of arbitrary cross-section, Ultrasonics 53 (7) (2013) 1227-1241.

[27] M. Mazzotti, I. Bartoli, A. Marzani, Ultrasonic leaky guided waves in fluid-coupled generic waveguides: hybrid finite-boundary element dispersion analysis and experimental validation, Journal of Applied Physics 115 (2014) 143512.

[28] T. Hayashi, D. Inoue, Calculation of leaky lamb waves with a semi-analytical finite element method, Ultrasonics 54 (6) (2014) 14601469.

[29] A. Pelat, S. Felix, V. Pagneux, On the use of leaky modes in open waveguides for the sound propagation modeling in street canyons, Journal of the Acoustical Society of America 126 (6) (2009) 2864-2872.

[30] B. Goursaud, Étude mathématique et numérique de guides d'ondes ouverts non uniformes, par approche modale (Mathematical and numerical study of non uniform open waveguides, modal approach, in French), Ph.D. thesis, École Polytechnique (2010).

[31] A.-S. Bonnet-BenDhia, B. Goursaud, C. Hazard, A. Prieto, Finite element computation of leaky modes in stratified waveguides, in: 5th Meeting of the Anglo-French-Research-Group, Vol. 128, 2009, pp. 73-86.

[32] F. Treyssède, K. L. Nguyen, A.-S. Bonnet-BenDhia, C. Hazard, On the use of a SAFE-PML technique for modeling two-dimensional open elastic waveguides, in: Acoustics 2012, France, April 23-27 2012, pp. 673-678.

[33] F. Treyssède, K. L. Nguyen, A.-S. Bonnet-BenDhia, C. Hazard, Finite element computation of trapped and leaky elastic waves in open stratified waveguides, Wave Motion 51 (2014) 1093 -1107.

[34] H. Gravenkamp, C. Birk, C. Song, Computational of dispersion curves for embedded waveguides using dashpot boundary condition, Journal of the Acoustical Society of America 135 (3) (2014) 1127- 1138.

[35] S. Kim, J. E. Pasciak, The computation of resonances in open systems using a perfectly matched layer, Mathematics of Computation 78 (267) (2009) $1375-1398$

[36] S. Kim, Cartesian PML approximation to resonances in open systems in $\mathbb{R}^{2}$, Applied Numerical Mathematics 81 (2014) 50-75.

[37] S. François, M. Schevenels, G. Lombaert, G. Degrande, A two-and-a-half-dimensional displacement-based PML for elastodynamic wave propagation, International Journal for Numerical Methods in Engineering 90 (7) (2012) 819 - 837.

[38] F. Tisseur, K. Meerbergen, The quadratic eigenvalue problem, SIAM Review 43 (2) (2001) 235-286.

[39] R. Lehoucq, D. C. Sorensen, C. Yang, ARPACK User's Guide: Solution of Large Scale Eigenvalue Problems with Implicitly Restarted Arnoldi Methods, SIAM, Philadelphia, PA, 1998.

[40] A. Bermúdez, L. Hervella-Nieto, A. Prieto, R. Rodríguez, An optimal perfectly matched layer with unbounded absorbing function for time-harmonic acoustic scattering problems, Journal of Computational Physics 223 (2) (2007) 469-488.

[41] E. A. Skelton, S. D. M. Adams, R. V. Craster, Guided elastic waves and perfectly matched layers, Wave Motion 44 (2007) 573-592.

[42] A. Pelat, S. Felix, V. Pagneux, A coupled modal-finite element method for the wave propagation modeling in irregular open waveguides, Journal of the Acoustical Society of America 129 (3) (2011) 1240-1249.

[43] A.-S. Bonnet-BenDhia, B. Goursaud, C. Hazard, A. Prieto, Finite element computation of leaky modes in stratified waveguides, in: Ultrasonic Wave Propagation in Non Homogeneous Media, Springer Proceedings in Physics, Vol. 128, 2009 , pp. 73-86. 
[44] Y. Ould Agha, F. Zolla, A. Nicolet, S. Guenneau, On the use of PML for the computation of leaky modes. An application to microstructured optical fibres, The International Journal for Computation and Mathematics in Electrical and Electronic Engineering 27 (1) (2008) 95-109.

[45] J. D. Achenbach, Wave Propagation in Elastic Solids, North-Holland, Amsterdam, 1973.

[46] S. Kim, J. E. Pasciak, Analysis of the spectrum of a cartesian perfectly matched layer (PML) approximation to acoustic scattering problems, Journal of Mathematical Analysis and Applications 361 (2) (2010) 420 -430.

[47] F. Treyssède, Mode propagation in curved waveguides and scattering by inhomogeneities: Application to the elastodynamics of helical structures, Journal of the Acoustical Society of America 129 (4) (2011) 1857-1868.

[48] A. Bernard, M. J. S. Lowe, M. Deschamps, Guided waves energy velocity in absorbing and non-absorbing plates, Journal of the Acoustical Society of America 110 (2001) 186-196.

[49] B. Pavlakovic, M. Lowe, Disperse User's Manual, Version 2.0.11, 131 p. (2001).

[50] S. Finneveden, Evaluation of modal density and group velocity by a finite element method, Journal of Sound and Vibration 273 (2004) $51-75$.

[51] J. M. Carcione, D. Gei, S. Treitel, The velocity of energy through a dissipative medium, Geophysics 75 (2) (2010) $37-47$.

[52] H. Cui, B. Zhang, S. Ji, Propagation characteristics of guided waves in a rod surrounded by an infinite solid medium, Acoustical Physics 56 (4) (2010) 412-421. 